\title{
Ecological Advanced Driver Assistance System for Optimal Energy Management in Electric Vehicles
}

\author{
Seyed Amin Sajadi-Alamdari ${ }^{1}$, Holger $\operatorname{Voos}^{1}$, and Mohamed Darouach ${ }^{2}$
}

\begin{abstract}
Battery Electric Vehicles have a high potential in modern transportation, however, they are facing limited cruising range. The driving style, the road geometries including slopes, curves, the static and dynamic traffic conditions such as speed limits and preceding vehicles have their share of energy consumption in the host electric vehicle. Optimal energy management based on a semi-autonomous ecological advanced driver assistance system can improve the longitudinal velocity regulation in a safe and energy-efficient driving strategy. The main contribution of this paper is the design of a real-time risksensitive nonlinear model predictive controller to plan the online cost-effective cruising velocity in a stochastic traffic environment. The basic idea is to measure the relevant states of the electric vehicle at runtime, and account for the road slopes, the upcoming curves, and the speed limit zones, as well as uncertainty in the preceding vehicle behaviour to determine the energy-efficient velocity profile. Closed-loop Entropic Value-at-Risk as a coherent risk measure is introduced to quantify the risk involved in the system constraints violation. The obtained simulation and field experimental results demonstrate the effectiveness of the proposed method for a semi-autonomous electric vehicle in terms of safe and energy-efficient states regulation and constraints satisfaction.
\end{abstract}

\section{INTRODUCTION}

$\mathbf{T}$ HE development of the Internal Combustion Engine (ICE) vehicle is clearly one of the most important achievements of modern technology for transport purposes. However, like in most other technologies, modern vehicle technology is also associated with its own challenges in safety, energy consumption and environmental contamination. The large number of ICE vehicles in use is leading to serious problems for the environment and human life around the world, and air pollution and global warming are problems of predominant concern. Therefore, it is well recognised that Battery Electric Vehicles (BEV) have one of the most promising powertrain technology for a sustainable future transportation [1].

\section{A. Background Information}

A BEV uses one or more electric motors for propulsion and electricity as the only source of propulsion energy. The BEV has relatively interesting features in comparison to other ICE

This work is supported by the FNR "Fonds national de la Recherche" (Luxembourg) through the AFR "Aides à la Formation-Recherche" Ph.D. grant scheme No. 7041503.

${ }^{1}$ Interdisciplinary Centre for Security, Reliability and Trust (SnT), University of Luxembourg, 29, avenue JF Kennedy, L-1855 Luxembourg. email: $\{$ amin.sajadi, holger.voos\}@uni.lu

${ }^{2}$ Centre de Recherche en Automatique de Nancy (CRAN) UMR-CNRS 7039, Université de Lorraine, IUT de Longwy, 186 rue de Lorraine, F-54400 Cosnes et Romain, France. e-mail: mohamed.darouach@univ-lorraine.fr vehicle alternatives. First of all, the efficiency of the BEV is far better than the ICE's efficiency. The BEV roughly have $80 \%$ to $95 \%$ efficiency while the ICE vehicles have $10 \%$ to $25 \%$ efficiency which means that only 10 to 25 units of energy are transferred to the wheels of the vehicle. Additionally, the BEVs offer the same or even better performance in comparison to the ICE vehicles, thanks to high torque at low speed of the electric motor. Furthermore, BEVs offer the opportunity to use different renewable energy resources. Despite the fact that also power plants have a contribution to carbon emissions, there are still different choices of getting true zero-emission electric from renewable energy resources. Introduction of the BEVs into the market has extended the opportunities for sustainable mobility and a new technological era which influences the driver behaviour patterns [2].

Environment-friendly BEVs are highly demanding for efficient utilisation of energy resources and reduction of energy consumption in road networks [3]. However, improvement of performance and energy efficiency is a challenging task where three energy conversion steps are generally investigated for the energy efficiency of the transportation. On the grid level, improvements could target at the grid-to-tank conversion that comprises the transfer of electric energy from the stationary distribution nodes of the grid to the onboard storage system. Considering the component or system control level, the tankto-wheel conversion of onboard energy to mechanical energy could be improved. Related reviews on the latest development in BEV technologies, impacts of BEV roll out and opportunities brought by BEV deployment are presented in [4]. Finally, the wheels-to-distance conversion considers the influence of the vehicle parameters and the driving strategy on the energy efficiency and is the main focus of this study.

A wide variety of factors such as the driving style, the BEV energy consumption characteristic map, its aerodynamic drag, the road slope with upcoming curves, traffic speed limits, the road visibility, dynamic of traffic flow, temperature, as well as weather conditions have a significant impact on the energy consumption of a BEV. Therefore, the goal of economical (eco-) driving is to adapt the driving strategy to an energyaware driving strategy. The Eco-driving is considered to be one of the most cost-effective methods in Intelligent Transportation System (ITS) to improve the road safety and energy efficiency of transportation. Improving the wheels-to-distance efficiency by controlling the driving profile reveals its potential when considering that it does not require structural changes to the system [5]. Eco-driving has the potential to enhance the capability of an automatic longitudinal control by minimising the energy consumption and emissions of the vehicle [6]. 
Encouraging drivers towards eco-driving can reduce energy consumption. In order to achieve an energy efficient drive strategy, a driver has to consider different factors such as the BEV dynamics, its energy consumption characteristic map, the road slopes, the curves, and the traffic situations in an anticipatory driving manner. However, drivers do not always and under all circumstances drive ecologically. Moreover, a mental focus on eco-driving might even lead to a distraction of the driver. On the other hand, utilising automatic Advanced Driver Assistance Systems (ADAS) can support drivers in various driving tasks. Herein, the anticipatory driving based Eco-ADAS can reduce the energy consumption by predicting earlier future situations. Several concepts attempt to implement the predictive Eco-driving in a more rigorous framework. In these concepts, the Eco-driving is regarded as an Optimal Control Problem (OCP) where the driving commands minimise the energy consumption for a given trip [5]. An overview on the different options to support the driver to reduce its energy consumption was provided in [7]. Model Predictive Control (MPC), also known as receding horizon optimal control, has been an attractive approach in comparison with alternative methods of multivariable control [8]. In MPC, the OCP is solved repeatedly in a receding horizon principle and the first element in a sequence of finite control actions is applied to the system at each sampling time.

Several works have been proposed in the literature to minimise the energy consumption of the vehicle. Speed advisory systems had been proposed in [9] for connected vehicles in order to minimise energy consumption over a planned route. In [9], the behaviour of the preceding vehicle was taken into account for a safe- and eco-driving system. Estimating and predicting traffic situations over time is an essential capability for sophisticated driver assistance systems and autonomous driving [10]. An efficient vehicle driving system, based on detailed anticipation of surrounding traffic with the aims of optimizing driving performance was proposed in [11]. A review of fundamental goals, development and future perspectives of driver assistance systems was provided in [12].

\section{B. Related Works in the Literature}

Cruise Control (CC), Adaptive Cruise Control (ACC) and Cooperative Adaptive Cruise Control (CACC) systems are the well-established ADAS that automate the throttle and brake control of the vehicle to retain the pre-set longitudinal velocity while maintaining a safe distance from the preceding vehicles. The vehicle under control of an Eco-ACC system minimises energy consumption in addition to other control objectives of the conventional ACC systems. Several contributions related to the application of predictive control in Eco-CC, Eco$\mathrm{ACC}$, and Eco-CACC systems can be found. For instance, a novel energy-efficient MPC was designed for the BEVs Eco-CC system by [13] and [14]. A linear real-time MPC to reduce the online computational burden by combining a move blocking strategy with a constraint-set compression strategy was introduced in [15].

Nonlinear Model Predictive Control (NMPC) is distinguished by the use of non-linear system models in the OCP to improve performance specifications (see e.g. [16]). An exemplary work of the NMPC, where an energy-efficient NMPC was introduced to drive a vehicle on roads with varying traffic and signals at intersections was introduced in [3]. An NMPC for a fuel-saving ACC system to improve the performance on tracking accuracy and fuel consumption by simultaneously considering the road elevation information and nonlinear powertrain dynamics was presented in [17]. An EcoACC system for plug-in hybrid electric vehicles to improve the total energy consumption and vehicle safety was introduced in [18]. Parametric uncertainties and exogenous disturbances are pervasive features of complex dynamical systems. Robust Model Predictive Control (RMPC) has been effectively utilised for systems with uncertainties (see e.g. [19]). An RMPC approach that regulates a minimum safe distance between vehicles taking into account the overall system delays and braking capacity of each vehicle was for instance proposed in [20].

In RMPC, the worst-case based design may lead to conservative control actions and low system performance. Stochastic Model Predictive Control (SMPC) has been introduced as a stochastic alternative to address the shortcomings of RMPC. The SMPC is based on the stochastic uncertainty of a process model and generally formulated as an expectation of the objective function with probabilistic constraints, so-called chanceconstraints (see e.g. [21]). Applied to an ACC systems, a scenario-based SMPC with driver behaviour learning capability for improving the powertrain performance was designed in [22]. A CACC system using stochastic, linear MPC strategies with the goal of minimising the fuel consumption in a carfollowing scenario was presented by [23]. Another example of a SMPC for the ACC and CACC systems under uncertainty based on the constant time gap policy were introduced in [24]. A real-time Stochastic Nonlinear Model Predictive Control (SNMPC) with probabilistic constraints and Risksensitive Nonlinear Model Predictive Control (RSNMPC) were presented in [25] and [26] to compute a safe and energyefficient cruising velocity profile online.

\section{Risk-sensitive Predictive Optimal Energy Management}

Although the conventional CC, ACC and CACC systems can assist the human driver to have a safe driving experience and improve the overall performance in an ITS, these are so far not capable of dealing with curvy roads and traffic signs information in an energy-efficient manner. In addition, even though the SNMPC has been introduced to improve the shortcoming of the SMPC and seems to be promising in terms of balancing conservatism in decision making and robustness to uncertainties, it has received relatively little attention in the literature so far, due to its limitation for realtime applications. Moreover, most of the mentioned SNMPCs are based on risk-neutral performance measures which may not be a suitable control strategy for the safety-critical ACC and CACC systems.

In order to achieve a sophisticated Eco-ADAS for application in ITS, it is required to develop an advanced EcoACC system with extended functionalities which is capable 
of operating on twisty roads rather than highways and dealing with a stochastic traffic environment. The main objective of this paper is to design an Eco-ACC platform with an advanced stochastic optimal control algorithm to meet the specific requirements of a semi-autonomous BEV to extend its cruising range. This paper presents an enhanced Eco-ACC system capable of dealing with road curvatures, speed limit zones, and uncertain behaviour of a preceding vehicle in a risk-sensitive manner. Herein, a real-time RSNMPC to plan the online safe and cost-effective cruising velocity with enhanced dynamic models is developed. The driving performance and energy efficiency depend on the accurate prediction of the deterministic parts such as the road geometry and statistically accurate anticipation of the stochastic parts of the system such as the uncertain preceding vehicle behaviour.

Based on the developed model, the BEV state information and plausible velocity profile of the preceding vehicle are propagated through the prediction horizon. The chanceconstraints evaluate the uncertain states trajectories. The main contribution of this paper is the introduction of a close-loop Entropic Value-at-Risk (EVaR) as a coherent risk measure to quantify the risk involved in the constraint violations. The inequality constraint handling method for the state inequality constraints are based on a semi-smooth transformation of Nonlinear Complementary Functions (NCF). The performance of the proposed concept in terms of real-time state regulation and constraint fulfilment is evaluated by simulation and field experimental tests. The achieved results demonstrate a significant improvement in energy consumption and safety of the BEV controlled by the RSNMPC.

The remainder of this paper is structured as follows: The system model is introduced in Section II. The RSNMPC formulation, risk management with inequality constraints are presented in Section III. Section IV includes a simulationbased evaluation as well as an experimental validation of the proposed concept in real driving tests, followed by the conclusion and description of future work in Section V.

\section{Notation}

Throughout this paper, $\mathbb{R}^{n}$ denotes the n-dimensional Euclidean space. $\mathbb{R}_{+}:=[0, \infty) . \mathbb{N}=\{1,2, \ldots\}$ is set of natural numbers. $\mathbb{N}_{+}:=\mathbb{N} \cup\{0\} . \mathbb{Z}_{[a, b]}:=\{a, a+1, \ldots, b\}$ is set of integers from $a$ to $b$. $\mathbf{E}$ denotes expectation and $\mathbf{E}_{x}[\cdot]:=\mathbf{E}[\cdot \mid x(0)=x]$ is the conditional expectation. $\mathbf{P r}$ denotes probability, and $\operatorname{Pr}_{x}[\cdot \mid x(0)=x]$ is the conditional probability distribution of random variable(s) $x$.

\section{SySTEM MODELS}

The fundamental Eco-ADAS concept proposed in this paper for a semi-autonomous BEV that extends the functionalities of an Eco-ACC system is presented in Fig. 1. Similar to the conventional ACC systems, the driver pre-sets the desired velocity with preferred safe distance from the preceding vehicle. The semi-autonomous Eco-ACC system predictively regulates the velocity with respect to the longitudinal motion of the host vehicle dynamics (BEV), its energy consumption, road geometry and traffic sign information, as well as the

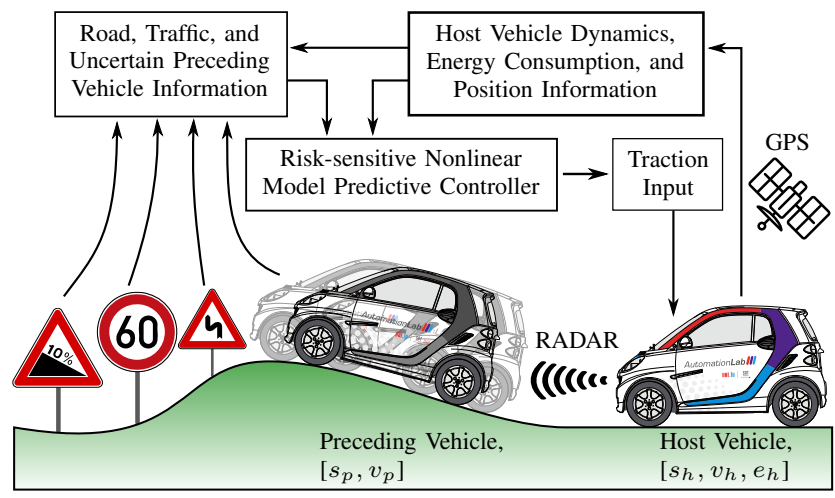

Fig. 1. Extended Eco-ACC Concept for a Semi-autonomous BEV

plausible motion of the preceding vehicle. While the driver still manually handles the steering control of the vehicle, this system should plan and realise a proper safe and energyefficient cruising velocity profile autonomously for the entire trip without requiring the driver's intervention. In addition, this system should be able to operate at full-range speed assistance and to handle cut-in/out scenarios.

\section{A. Vehicle Dynamics}

The electric propulsion subsystems of the BEV include vehicle traction control inputs (throttle and brake pedals), a power electronic converter, an electric machine, a generally single-gear mechanical transmission, and the driving wheels. The energy source subsystem involves a battery package, an energy management-monitor unit, and an energy recharging entity. The auxiliary subsystem consists of the power steering, the cabin climate control, and the auxiliary supply units (for more details, see [1]).

The position $\left(s_{h}\right)$ and velocity $\left(v_{h}\right)$ along the longitudinal motion of the BEV can be expressed by Newton's second law of motion, where the vehicle is assumed to be a point mass at the centre of gravity as follows:

$$
\begin{aligned}
& \dot{s}_{h}=v_{h}, \\
& \dot{v}_{h}=\left(F_{\text {trac }}-F_{\text {res }}\right) / M .
\end{aligned}
$$

Herein, $M, F_{\text {trac }}(t)$, and $F_{\text {res }}(t)$ are the equivalent mass of the vehicle, the traction force, and the total motion resistive forces, respectively. The equivalent mass can be calculated by an empirical relation as $M=m\left(1+\delta_{1}+\delta_{2} i_{g}^{2}\right)$, where $m$ is the kerb mass of the vehicle, $\delta_{1}$ represents the total angular inertial moment of the wheels, $\delta_{2}$ represents the effect of the powerplant-associated rotating parts, and $i_{g}$ is the single transmission ratio [1].

The traction force depends on the equivalent mass and control input as $F_{\text {trac }}(t):=M u(t)$. The control input is bounded by the physical limits of the traction force that the wheelroad contact can support without slip $\left(u_{\min }\left(v_{h}\right) \leq u(t) \leq\right.$ $\left.u_{\max }\left(v_{h}\right)\right)$ [1]. The main total resistive force $\left(F_{\text {res }}\right)$ including 
aerodynamic drag force $\left(F_{a r d}\right)$, gradient force $\left(F_{g r d}\right)$, and rolling resistance force $\left(F_{r r}\right)$ is represented by:

$$
\begin{aligned}
F_{r e s} & =F_{\text {ard }}+F_{\text {grd }}+F_{r r}, \\
F_{\text {ard }} & =\frac{1}{2} \rho A_{f} C_{D}(d) v_{h}^{2}, \\
F_{\text {grd }} & =M g \sin \left(\theta\left(s_{h}\right)\right), \\
F_{r r} & =C_{r r}\left(v_{h}\right) M g \cos \left(\theta\left(s_{h}\right)\right),
\end{aligned}
$$

where $\rho, A_{f}, g, \theta\left(s_{h}\right)$, and $C_{r r}\left(v_{h}\right)$ are the air density, the vehicle frontal area, the gravitational acceleration, the road slope angle as a function of the host vehicle position, and the velocity dependent rolling resistance coefficient, subsequently. The rolling resistance coefficient for passenger vehicles on a concrete road can be approximated as $C_{r r}(v)=0.01(1+$ $v / 576)$ [1]. The $C_{D}(d)$ is the aerodynamic drag coefficient that depends on the nominal aerodynamic drag coefficient, $C_{D 0}$, and the relative distance between the preceding and host vehicles, $d=s_{p}-s_{h}$. Vehicle drag reductions arise from close spacing with the preceding vehicle [27].

\section{B. Road Geometry and Static Traffic Models}

For any Eco-ADAS corresponding to safety and energy management applications it is advantageous to include more detailed information about the road geometries and static traffic regulations. The road slopes, road curves, and traffic speed limit zone data are modelled as continuous and differentiable functions in [28]. In that method, the road slope profile $\left(f_{s l p}(\theta(s))\right)$ is proposed to be the sum of quadratic functions of the vehicle's position representing each road segment's slope data as follows:

$$
f_{s l p}(\theta(s)):=\sum_{n=1}^{N_{s g m}} H_{\left(s-s_{n-1}\right)}^{n}\left(a_{n} s^{2}+b_{n} s+c_{n}\right) H_{\left(s-s_{n}\right)}^{n},
$$

where $N_{s g m}$ is the number of road segments, $H_{\left(s-s_{n-1}\right)}^{n}$ and $H_{\left(s-s_{n}\right)}^{n}$ are hyper-functions of the $n^{t h}$ road segment. These functions represent the data points in each segment of the road utilising the hyper-function concept to interconnect the estimated segments of the road at the positions, $s_{n-1}$ and $s_{n}$ of the boundaries. The hyper-functions may be represented by the approximate Heaviside's functions at the boundary position values $s_{n-1}$ and $s_{n}$.

The road curves and profiles of the traffic speed limits are modelled in a similar way [28]. The resulting curve $f_{\text {crv }}(\delta(s))$ that is used to express the overall road curve profile is obtained as:

$$
f_{c r v}(\delta(s)):=\sum_{n=1}^{N_{c r v}} H_{\left(s-s_{e n t}\right)}^{n}\left|\frac{1}{R_{c r v_{n}}(s)}\right| H_{\left(s-s_{e x t}\right)}^{n},
$$

where $N_{c r v}$ is the number of road curves, and $R_{c r v_{n}}$ is the radius of a circle valid for the curve's arc length with two position points, $s_{\text {ent }}$ and $s_{\text {ext }}$, at the respective entrance and exit positions. The $R_{c r v_{n}}(\cdot)$ for a straight road segment can be considered as a large numerical value. Furthermore, the traffic speed limit profile $\left(f_{l m t}(s)\right)$ can be modelled as:

$$
f_{l m t}(s):=\sum_{n=1}^{N_{l m t}} H_{\left(s-s_{s t r}\right)}^{n}\left(v_{l m t}-v_{\text {max }}\right) H_{\left(s-s_{\text {end }}\right)}^{n}+v_{\text {max }},
$$

where $N_{l m t}$ is the number of speed limit zones, and $v_{l m t}$ is the specified speed limit value at positions starting from $s_{s t r}$ up to the end of the zone $s_{\text {end }}$. The velocity $v_{\max }$ is the maximum speed value of the host vehicle. This method to model the road geometry and static traffic data improve the trade-off challenge between model complexity and accuracy (high and low-fidelity models) for the Eco-ADAS application [25], [28].

\section{Energy Consumption Dynamics}

Energy consumption of a BEV depends on a number of factors including the driven velocity, the acceleration profile, geometric characteristics of roads, and traffic situations. The energy consumption could have a wide variation depending on different operating points of the electric machine [1]. For a given velocity at a given traction force, the operating point of the electric machine and the related power consumption or regeneration could be determined [28].

The power consumption during cruising at constant speed is equal to the resistive power. This can be approximated through a curve-fit process of measured data by a polynomial of the velocity in the form $f_{\text {cruise }}(v)=b_{3} v_{h}^{3}+b_{2} v_{h}^{2}+b_{1} v_{h}+b_{0}$ (for more details, see [3] and [28]). The acceleration of the vehicle considering only the regenerative energy zone in the hybrid (regenerative and friction) brake system can be approximated by a similar process with measured data using a polynomial of the control input as $f_{a}(u)=a_{2} u^{2}+a_{1} u+a_{0}$. Therefore, at any given velocity and control input, a linear relation of the traction power-to-mass ratio can describe the energy consumption of the BEV as:

$$
\dot{e}_{h}=f_{a}(u)\left(p_{\text {trac }} / M\right)+f_{\text {cruise }}(v),
$$

where $p_{\text {trac }}$ denotes the traction power. This model is capable of capturing the full-range energy consumption of a BEV based on the velocity and the control input [28].

Fig. 2 shows the power consumption model of a Smart Fortwo Electric Drive (Smart-ED) commercial BEV based on traction input and velocity. Each contour line represents the related power consumption (in $k W$ ). At the higher traction input and velocity, a positive amount of energy is consumed at a higher rate. In contrast, in regenerative braking zones at different velocity, a limited amount of energy can be recovered. This novel model is capable of representing the regenerative braking effect for the full-range velocity and traction input limits. This way, the power consumption of the BEV can be estimated by modelling a traction-velocity characteristics map of the electric machine. Considering equation (10) and the measured data from a two-axles dynamometer test with the Smart-ED, the proposed model for the energy consumption is approximated through the curve-fit process with $98.46 \%$ coefficient of determination (R-squared). 


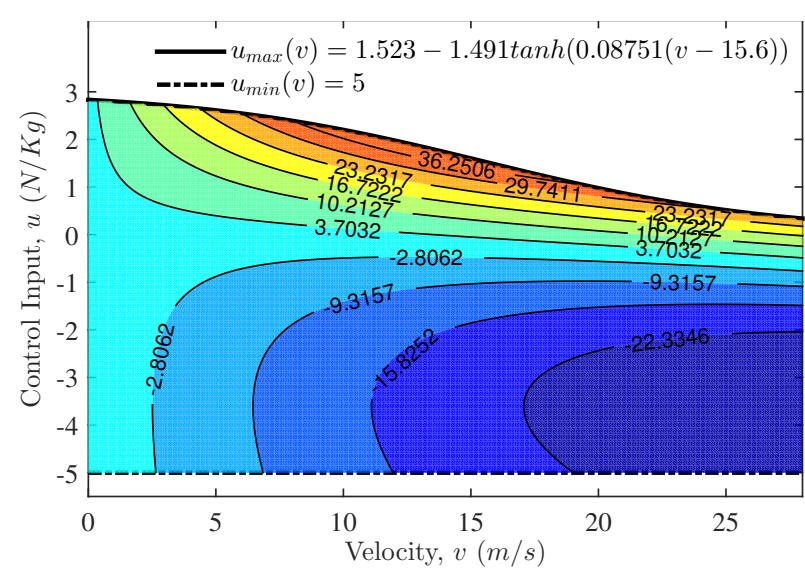

Fig. 2. Power consumption of the Smart-ED [28]

\section{Preceding Vehicle Physical-Statistical Motion Model}

Knowledge representation of dynamic traffic including a prediction model of a plausible motion of the preceding vehicles improves the performance of decision-making processes in Eco-ADAS applications. However, high entropy in the traffic system leads to a challenging task to derive a computationally efficient and tractable model. Research related to anticipating the possible trajectory of the preceding vehicle into the near/far-term future has a long track in the ADAS applications. For instance, a nominal case used for the prediction model of the preceding vehicle (constant velocity), where any change in the predicted velocity is considered as a disturbance on the system. A Markov chain model with the driver behaviour learning algorithm was proposed in [22]. A sigmoid-based function to estimate states of the preceding vehicle within the prediction horizon was introduced in [3]. A stochastic prediction method using Bayesian networks utilised for near-term future prediction was presented in [23].

Although the proposed methods mentioned in works of literature are effective for near-term prediction, rapid divergence can be experienced in far-term future prediction. A physicalstatistical motion model of the preceding vehicle robust to far-term future prediction was developed in [25] and [26]. The proposed model is based on the $85^{\text {th }}$ percentile speed concept and road geometry information. The $85^{\text {th }}$ percentile speed is referred to as spot speed study, defined as the speed at or below which $85^{\text {th }}$ percent of vehicles travel a given location based on free-flowing conditions over a time period [29]. The free-flowing conditions refer to the motion of the preceding vehicle that has at least three seconds time headway. In addition, other factors such as road slope profile and traffic speed limit zones information can be considered to estimate a more accurate velocity trajectory. The preceding vehicle position $\left(s_{p}\right)$ and velocity $\left(v_{p}\right)$ generally can be measured by RAdio Detection And Ranging (RADAR) in the ACC systems. Thus, the introduced dynamic model to propagate the preceding vehicle position and velocity at time $t$ can be

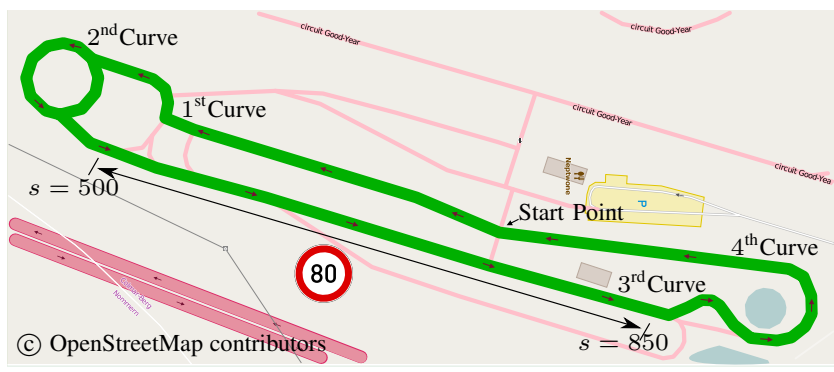

Fig. 3. Test track, Centre de Formation pour Conducteurs [30]

determined as follows:

$$
\begin{aligned}
& \dot{s}_{p}:=v_{p}, \\
& \dot{v}_{p}:=X_{85^{t h}}\left(1-\left(\frac{v_{p}}{f_{85^{t h}}}\right)^{4}-\frac{\sin \left(f_{s l p}\left(\theta\left(s_{p}\right)\right)\right)}{\sin \left(\frac{\pi}{4}\right)}\right), \\
& f_{85^{t h}}:=\min \left\{\omega_{85^{t h}} v_{85^{t h}}\left(f_{c r v}\left(\delta\left(s_{p}\right)\right)\right), f_{l m t}\left(s_{p}\right)\right\} \\
& v_{85^{t h}}\left(\delta\left(s_{p}\right)\right):=m_{1} \exp ^{\left(-m_{2} \delta\left(s_{p}\right)\right)}+m_{3} \exp ^{\left(-m_{4} \delta\left(s_{p}\right)\right)},
\end{aligned}
$$

where $X_{85^{t h}}$ is the acceleration of the preceding vehicle at $85^{t h}$ percentile assumed to lie in a normal distribution i.i.d. $X \sim \mathcal{N}\left(\mu_{p}, \sigma_{p}\right)$ with the mean $\mu_{p}$ and variance $\sigma_{p}^{2}$. The $\omega_{85^{t h}}, m_{1}, \ldots, m_{4}$ are tunable positive constants. The position based function $v_{85^{t h}}(\cdot)$, represents the $85^{t h}$ percentile curve speed of the vehicles along the road curves with statistical data adapted from [29]. To conclude, the introduced model is continuous and differentiable that is capable of propagating a plausible trajectory for the preceding vehicle motion along the prediction horizon (for more details, see [25] and [26]).

The introduced physical-statistical motion model for the preceding vehicle behaviour prediction has been evaluated on a closed test track located at Colmar-Berg, Luxembourg (Fig. 3) [25]. This test track has a total length of $1.255 \mathrm{~km}$ and includes curves, a speed limit zone with relative slope profile. This track has four main curves including $\mathrm{crv}_{1}=20 \mathrm{~m}$, $c r v_{2}=25 \mathrm{~m}, \mathrm{crv}_{3}=15 \mathrm{~m}$, and $\mathrm{crv}_{4}=27 \mathrm{~m}$ radius. The straight road segments are considered to have a nearly infinite radius. In addition, a speed limit $v_{l m t}=13.89 \mathrm{~m} / \mathrm{s}$ zone is assumed between positions $500 \leq s \leq 850$. The test track slope profile, $f_{s l p}(\theta(s))$, is fitted within nine segments with 98.93\% coefficient of determination [28]. Fig. 4 demonstrates the preceding vehicle motion prediction based on the $85^{\text {th }}$ percentile speed concept considering the test track geometry and speed limit zone information [25].

The measured data include seven different velocity profiles of human drivers on the test track. The physical-statistical motion model performance to foresee an expected velocity profile based on road and traffic information demonstrate its capability to anticipate the position and velocity of the preceding vehicle without feedback measurement updates. Significant statistical accuracy can be shown in term of the median and the related variations from the practical experiments obtained by the human drivers (H-\#) and the proposed physical-statistical motion model (PS-M) on the test track. The average velocity of all human drivers is $11.68 \mathrm{~m} / \mathrm{s}$, and the average predicted velocity of the physical-statistical motion model is $12.26 \mathrm{~m} / \mathrm{s}$. 

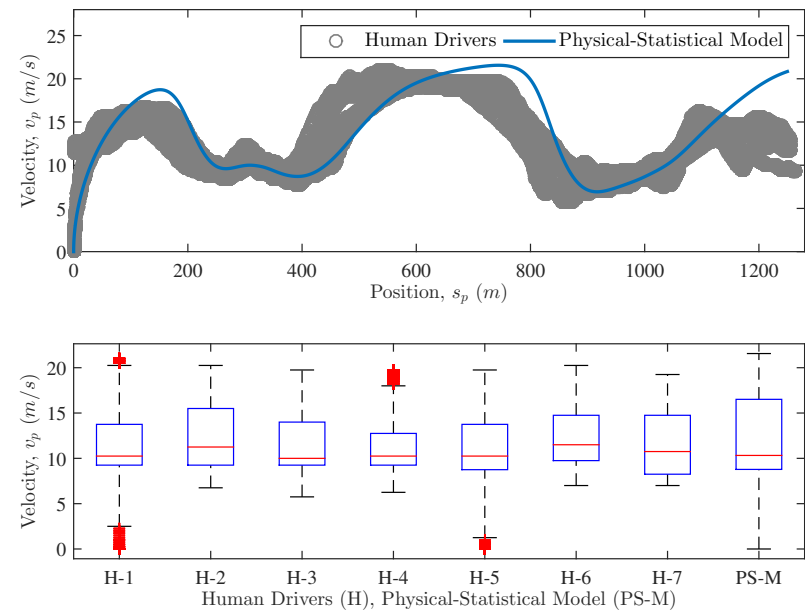

Fig. 4. Open-loop preceding vehicle velocity prediction with statistical results [25]

\section{Risk-SEnsitive Nonlinear PREdictive Control}

For the sake of completeness, a general SNMPC formulation and Entropic Value-at-Risk (EVaR) as a coherent risk measure will be reviewed. The proposed risk-averse certainty equivalent reformulation of the SNMPC based on minimum principle with a close-loop inequality constraint handling method will be introduced. In addition, the risk-averse certainty equivalent reformulation of the RSNMPC with its application for the EcoACC system will be presented.

\section{A. Stochastic Nonlinear Model Predictive Control (SNMPC)}

Consider a general stochastic, discrete-time system:

$$
x_{t+1}=f\left(x_{t}, u_{t}, \omega_{t}\right),
$$

where $t \in \mathbb{N}_{+} ; x_{t} \in \mathbb{R}^{n_{x}}$ is the system states vector and $u_{t} \in \mathbb{U} \subset \mathbb{R}^{n_{u}}$ is a non-empty measurable set for the inputs. $\omega_{t} \in \mathbb{R}^{n_{\omega}}$ is disturbances vector that is unknown at the current and future time instants. The $\omega_{t}$ is composed of i.i.d. random variables within the known sample space $\Omega$, the set of events ( $\sigma$-algebra) $\mathcal{F}$, and the allocations of probabilities, $\mathcal{P}$ to events (exogenous information). The $f(\cdot)$ is a nonlinear Borel-measurable vector of functions that describes the system dynamics [21].

Let $N \in \mathbb{N}$ be the both state and control prediction horizon. Define an $N$-stage feedback control policy as:

$$
\boldsymbol{\pi}:=\left\{\pi_{0}(\cdot), \pi_{1}(\cdot), \ldots, \pi_{N-1}(\cdot)\right\},
$$

where the Borel-measurable function $\pi_{i}(\cdot): \mathbb{R}^{(i+1) n_{x}} \rightarrow \mathbb{U}$, for all $i=0, \ldots, N-1$ is a general state feedback control law [21]. The control input $u_{i}$ is selected as the feedback control law $u_{i}=\pi_{i}(\cdot)$ at the $i^{t h}$ stage of the control policy. In receding horizon optimal control, the cost function of the OCP is commonly defined as:

$$
V_{N}\left(x_{t}, \boldsymbol{\pi}\right):=\mathbf{E}_{x_{t}}\left[\sum_{i=0}^{N-1} J_{c}\left(\hat{x}_{i}, u_{i}\right)+J_{f}\left(\hat{x}_{N}\right)\right],
$$

where $J_{c}: \mathbb{R}^{n_{x}} \times \mathbb{U} \rightarrow \mathbb{R}_{+}$and $J_{f}: \mathbb{R}^{n_{x}} \rightarrow \mathbb{R}_{+}$are the costper-stage function and the final cost function, respectively. The $\hat{x}_{i}$ denotes the predicted states at time $i$ given the initial states $\hat{x}_{0}=x_{t}$, control law $\left\{\pi_{i}(\cdot)\right\}_{i=0}^{i-1}$, and disturbance realizations $\left\{\omega_{i}\right\}_{i=0}^{i-1}[21]$.

A general form of chance-constraints is defined by:

$$
\mathbf{P r}_{x_{t}}\left[g_{j}\left(\hat{x}_{i}\right) \leq 0\right] \geq \beta_{j}, \quad \text { for all } j \in \mathbb{Z}_{[1, s]}, i \in \mathbb{Z}_{[1, N]},
$$

where $g_{j}: \mathbb{R}^{n_{x}} \rightarrow \mathbb{R}$ is a Borel-measurable function, $s$ is the total number of inequality constraints, and $\beta_{j} \in(0,1)$ denotes the lower bound for the probability $g_{j}\left(\hat{x}_{i}\right) \leq 0$ that needs to be satisfied. Different probability levels $\beta_{j}$ are assigned for different inequality constraints. The conditional probability $\mathbf{P r}_{x_{t}}$ indicates the probability of $g_{j}\left(\hat{x}_{i}\right) \leq 0$ holds based on initial states $\hat{x}_{0}=x_{t}$; please note that the predicted states $\hat{x}_{i}$ depend on disturbances $\left\{\omega_{i}\right\}_{i=0}^{i-1}$ [21].

Using the cost function (17) and the individual chanceconstraint (18), the OCP for (15) is formulated as follows:

$$
V_{N}^{*}\left(x_{t}\right):=\underset{\pi}{\operatorname{minimise}} \quad V_{N}\left(x_{t}, \boldsymbol{\pi}\right)
$$

subject to:

$\hat{x}_{i+1}=f\left(\hat{x}_{i}, \pi_{i}, \omega_{i}\right), \quad$ for all $i \in \mathbb{Z}_{[0, N-1]}$,

$\pi_{i}(\cdot) \in U$,

for all $i \in \mathbb{Z}_{[0, N-1]}$,

$\mathbf{P r}_{x_{t}}\left[g_{j}\left(\hat{x}_{i}\right) \leq 0\right] \geq \beta_{j}, \quad$ for all $j \in \mathbb{Z}_{[1, s]}, i \in \mathbb{Z}_{[1, N]}$,

$\omega_{i}=(\Omega, \mathcal{F}, \mathcal{P})$

for all $i \in \mathbb{Z}_{[0, N-1]}$,

$\hat{x}_{0}=x_{t}$,

where $V_{N}^{*}\left(x_{t}\right)$ denotes the optimal value function under the optimal control policy $\pi^{*}$. The OCP in receding horizon principle involves applying the first element of the control action sequence $u_{t}=\pi_{0}^{*}(\cdot)$ repeatedly to the system at each sampling time.

Generally there is no exact solution to the stochastic OCP (19) due to i) the arbitrary form of the feedback control laws; ii) the nonconvexity and general intractability of chance constraints; iii) the computational complexity associated with uncertainty propagation through complex system dynamics; iv) the risk-neutral expectation assessment of future random outcomes for safety-critical systems where one desires to regulate the control actions so that they are robust enough to uncertainties [31].

The next subsection presents the EVaR concept as an openloop assessment of the chance-constraint. In this paper, closedloop EVaR chance-constraint evaluation is introduced as the proposed contribution of the subsection.

\section{B. Closed-loop Entropic Value-at-Risk (EVaR)}

Several approximations have been developed to obtain a feasible solution rather than an exact solution for the OCP (19). In this section, the challenges ii) and iv) are addressed for solving approximately the stochastic OCP. An alternative objective to risk-neutral (17) can be a control policy sensitive to nonlinear risk defined as:

$$
\kappa_{\rho}\left(V_{N}\left(x_{t}, \boldsymbol{\pi}\right)\right):=\rho^{-1} \log \mathbf{E}_{x_{t}}\left[\exp { }^{\left(\rho V_{N}\left(x_{t}, \boldsymbol{\pi}\right)\right)}\right],
$$

where $\rho \in \mathbb{R} \backslash\{0\}$ is a risk-sensitivity parameter that determines the controller's attitude toward uncertainty: $\rho<0$ 
indicates risk-seeking and $\rho>0$ implies risk-averse policy [31]. Generally, it is straightforward to find Taylor series expansion around the point $\rho=0$, where it was usual to quantify risk in terms of the variance [32]. This leads to various forms of stochastic OCPs such as the well-known Markowitz mean-variance approach. However, the current more sophisticated paradigm for risk measurement, mainly interested in downside risk, goes beyond the variance [32]. In addition, the (20) also known as entropic risk measure with parameter $\rho$ which can be represented by mean of a coherent risk measure that is computationally tractable if the objective function can be computed efficiently [33].

A coherent risk measure satisfies the transitional invariance, sub-additivity, monotonicity, and positive homogeneity properties. The Value-at-Risk (VaR) and Conditional VaR (CVaR) are the most popular and widely used risk measurements. The VaR and CVaR intuitively evaluate the expectation and conditional expectation of (18) respectively on a tail part of its distribution ( $\beta_{j}$-percentile). However, the VaR does not satisfy the sub-additivity property while CVaR cannot be computed efficiently. In order to address these limitations, the coherent Entropic Value-at-Risk (EVaR) has been recently introduced [33]. The EVaR provides the tightest upper bound one can find using the Chernoff inequality for the VaR and CVaR with the same confidence levels [33]. The EVaR with confident level $\left(\beta_{j}=1-\alpha_{j}\right)$ is defined as follows:

$$
E V a R_{1-\alpha_{j}}\left(g_{j}\left(\hat{x}_{i}\right)\right):=\inf _{z>0}\left\{z^{-1} \ln \left(M_{g_{j}\left(\hat{x}_{i}\right)}(z) / \alpha_{j}\right)\right\},
$$

where $M_{g_{j}\left(\hat{x}_{i}\right)}=\mathbf{E}_{x_{t}}\left[\exp ^{\left(z g_{j}\left(\hat{x}_{i}\right)\right)}\right]$ is the moment-generating function of $g_{j}\left(\hat{x}_{i}\right)$.

The properties of coherent the risk measure have intuitive interpretations in the financial industry, which can be extended to energy management systems [32]. In the case of the Eco-ACC system, for instance, the relative distance can be interpreted as a portfolio of energy consumption and travel time. The higher risk of rear-end collision cause closer car following situations with shorter travel time (higher probability of constraint violation). The lower risk, on the other hand, leads to a longer travel time (lower expected reward) with a lower risk of rear-end collision or constraint violation. The motivation for the risk-sensitive optimal controller is to find a tradeoff between the expected profit (desired pre-set velocity tracking) and the risk. One may minimise the OCP given by (19) based on approximate coherent risk measure EVaR or minimise the OCP with the risk-sensitive cost function (20). Although the solution of both approaches is not necessarily equivalent, it is possible to obtain similar results by properly tuning the risk-sensitivity parameter [32].

In the case of the risk-averse control policy, the proposed closed-loop confidence level $\left\{\beta_{i}(t)\right\}_{m-1}^{0}$ is estimated based on a Two-pass algorithm to compute the standard deviation using the Exponential Moving Average of the past $\left\{p\left(t_{m-1}\right), p\left(t_{m-2}\right), \ldots, p(0)\right\} M$-measurement vector. In this method, the samples moving average is calculated by:

$$
\bar{p}=\frac{\sum_{j=0}^{m-1} x_{j}}{M} .
$$

Afterwards, the unbiased estimation for the variance of samples can be computed based on the Bessel's correction given by:

$$
\operatorname{Var}(P):=\sigma^{2}=\frac{\sum_{i=0}^{m-1}\left(p_{i}-\bar{p}\right)^{2}}{M-1}
$$

where $\sigma$ is the corrected sample standard deviation ( $\sigma=$ $\sqrt{\operatorname{Var}(P)})$. This algorithm is numerically stable if $M$ is small (for more details see [34]). Generally, the standard deviation is considered as a tuning parameter in works of literature. A larger value results in conservative but robust behaviour while a small value could lead to high performance but more frequent constraints violation. However, the proposed method to estimate the standard deviation utilises the advantages of feedback to reduce the conservative behaviours of the riskaverse chance-constraints and improves the trade-off between the performance and robustness.

The next subsection presents the minimum principle with a real-time numerical method to solve the OCPs. The certainty equivalent control policy based on rolling disturbance estimation is the proposed contribution in the following subsection.

\section{Risk-averse Certainty Equivalent Minimum Principle}

In this subsection, the challenges i) and iii) are addressed for solving approximately the stochastic OCP given by (19). The main idea is based on a suboptimal control policy socalled certainty equivalence principle with rolling disturbance estimation. In this method $\hat{\omega}_{i}$ is interpreted as the prediction of expected disturbance values, $\hat{\omega}_{i}=\mathbf{E}\left[\omega_{i}\right]$, for the uncertainty propagation. Hence, the proposed method emphasizes on early detection and reduction of large recourse, rather than the compensation of non-optimal decisions. The $\hat{\omega}_{i}$ generally might be obtained by various methods such as conditional expectations, statistical models, etc. The expected disturbance may also be approximated by continuous dynamics. Consequently, the system function (15) can be rewritten as deterministic surrogate form as:

$$
\bar{x}_{t+1}=\bar{f}\left(\bar{x}_{t}, u_{t}\right) \text {, }
$$

where $\hat{\bar{x}}_{t} \in \mathbb{R}^{n_{x}+n_{\omega}}$ denotes the predicted nominal states including auxiliary states $\hat{\omega}_{i}$. The i.i.d random variables assumption of the $\omega_{i}$ is no longer required. Therefore, the stochastic OCP cost function defined by (17) reduces to certainty equivalent form as:

$$
V_{N}\left(\bar{x}_{t}, \boldsymbol{\pi}\right):=\sum_{i=0}^{N-1} J_{c}\left(\hat{\bar{x}}_{i}, u_{i}\right)+J_{f}\left(\hat{\bar{x}}_{N}\right),
$$

where $J_{c}: \mathbb{R}^{n_{x}+n_{\omega}} \times \mathbb{U} \rightarrow \mathbb{R}_{+}$and $J_{f}: \mathbb{R}^{n_{x}+n_{\omega}} \rightarrow \mathbb{R}_{+}$.

The risk-averse certainty equivalent OCP can be obtained by substituting the Eq. (17) with (25), the Eq. (15) with (24) 
and the chance-constraints given by (18) with its EVaR upper bound Eq. (21) based on initial nominal states $\hat{\bar{x}}_{0}=\bar{x}_{t}$.

$$
V_{N}^{*}\left(\bar{x}_{t}\right):=\underset{\pi}{\operatorname{minimise}} \quad V_{N}\left(\bar{x}_{t}, \boldsymbol{\pi}\right)
$$

subject to:

$\hat{\bar{x}}_{i+1}=f\left(\hat{\bar{x}}_{i}, \pi_{i}\right), \quad$ for all $i \in \mathbb{Z}_{[0, N-1]}$,

$\pi_{i}(\cdot) \in U$,

for all $i \in \mathbb{Z}_{[0, N-1]}$,

$E V a R_{1-\alpha_{j}}\left(g_{j}\left(\hat{\bar{x}}_{i}\right)\right) \leq 0$, for all $j \in \mathbb{Z}_{[1, s]}, i \in \mathbb{Z}_{[1, N]}$,

$\hat{\bar{x}}_{0}=x_{t}$.

It is noteworthy that the obtained certainty equivalent policy is a quite computationally efficient strategy, while accounts the effects of system uncertainty or risk association with the planning of future control actions.

Numerical methods to solve the OCPs are generally classified into three main categories. Dynamic Programming (DP) breaks the problem into smaller sub-problems. It is based on Bellman's principle of optimality to propagate the cost-perstage function. This approach generally leads to the HamiltonJacobi-Bellman (HJB) equation where it is mainly solved backwards in time, from the end of prediction horizon $t=N$ to the beginning $t=0$. Due to the curse of dimensionality, the HJB is in general computationally expensive and is only applicable to systems with low dimensions. An alternative approach is Direct methods that are based on the numerical solution of a finite dimensional OCP which corresponds to a discrete approximation of the original continuous-time OCP. The solution interval is divided into smaller intervals which the initial value problem in each smaller intervals is solved. A matching condition forms the final solution on the entire interval.

In addition, Indirect methods based on Pontryagin's Minimum Principle (PMP) relate closely to the Dynamic Programming (DP) scheme, but they are more efficient in solving the OCP which satisfy the necessary conditions for the optimality. The achieved nonlinear Two-Point Boundary-Value Problem (TP-BVP) can then be solved numerically. The closed-loop OCP for Mechatronic systems controlled with a sampling period in the order of milliseconds leads to a TP-BVP in receding horizon control principle which need to be solved in real-time. Therefore, this study focuses on the indirect methods based on PMP for the real-time OCP.

Let's consider the achieved surrogate dynamic of the system (24) with initial nominal states. The constraints on the system dynamics can be adjoined to the Lagrangian $J_{c}(\cdot)$ by introducing the time-varying Lagrange multiplier vector $\lambda \in \mathbb{R}^{n_{x}}$, where its elements are also known as the co-states of the system. Equality constraints can also be imposed over the prediction horizon. This motivates the construction of the Hamiltonian $(H)$ defined as Lagrangian duality as follows:

$$
H(\bar{x}, u, \lambda, \mu):=J_{c}(\bar{x}, u)+\lambda^{T} f(\bar{x}, u)+\mu^{T} C(\bar{x}, u),
$$

where $\lambda^{T}$ denotes the transpose of $\lambda$ and $\mu$ is Lagrange multipliers of equality constraints. The $C(\cdot) \in \mathbb{R}^{n_{c}}$ is the equivalent vector-valued equality constraints function. Reformulating the Lagrangian as a Hamiltonian, in which case the solutions are local minima for the Hamiltonian is known as Pontryagin's
Minimum Principle (PMP). The solution can be global minima if the (27) have convex structure.

The first-order necessary conditions for the optimal state trajectory $\bar{x}^{*}$, optimal control input $u^{*}$, corresponding costate multiplier vector $\lambda^{*}$, and optimal Lagrange multipliers $\mu^{*}$ should satisfy:

$$
\begin{aligned}
& H_{u}\left(\bar{x}^{*}(t), u^{*}(t), \lambda^{*}(t), \mu^{*}(t)\right)=0, \\
& \dot{\lambda}^{*}(t)=-H_{\bar{x}}^{T}\left(\bar{x}^{*}(t), u^{*}(t), \lambda^{*}(t), \mu^{*}(t)\right), \\
& \lambda_{N}^{*}(t)=J_{f_{\bar{x}}}\left(\bar{x}_{N}^{*}(t)\right),
\end{aligned}
$$

where $H_{u}$ and $H_{\bar{x}}$ are the Jacobian matrix of Hamiltonian with respect to the control inputs and system states, respectively. Let's define a vector of the control inputs and Lagrange multipliers as follows:

$$
U(t):=\left[u_{0}^{*^{T}}(t), \mu_{0}^{*^{T}}(t), \ldots, u_{N-1}^{*^{T}}(t), \mu_{N-1}^{*^{T}}(t)\right] \in \mathbb{R}^{n N},
$$

where $n:=n_{u}+n_{c}$. Considering the initial condition, (24), (28), and equivalent equality constraints, the necessary optimality conditions can be regarded as one equation as follows:

$$
F(U, \bar{x}, t):=\left[\begin{array}{c}
H_{u}^{T}\left(\bar{x}_{0}^{*}(t), u_{0}^{*}(t), \lambda_{1}^{*}(t), \mu_{0}^{*}(t)\right) \\
C\left(\bar{x}_{0}^{*}(t), u_{0}^{*}(t)\right) \\
\vdots \\
H_{u}^{T}\left(\bar{x}_{N-1}^{*}(t), u_{N-1}^{*}(t), \lambda_{N}^{*}(t), \mu_{N-1}^{*}(t)\right) \\
C\left(\bar{x}_{N-1}^{*}(t), u_{N-1}^{*}(t)\right)
\end{array}\right]=0 .
$$

Generally one may solve the (30) using costly iterative methods such as Newton's methods. However, solution of the (30) can be obtained utilising the Continuation and Generalized Minimal RESidual (C/GMRES) method proposed in [35]. The main idea is based on choice of $U(0)$ so that $F(U(0), \bar{x}(0), 0)=0$ and determine $\dot{U}$ such that:

$$
\dot{F}(U, x, t)=A_{s} F(U, x, t),
$$

where $A_{s}$ is a stable matrix introduced to stabilise $F(\cdot)=0$. If $F_{U}$ is nonsingular, a differential equation for $U$ can be written as:

$$
\dot{F}(U, \bar{x}, t)=F_{U}^{-1}\left(A_{s} F-F_{\bar{x}} \dot{\bar{x}}-F_{t}\right),
$$

which can be regarded as a linear algebraic equation with a coefficient matrix $F_{U}$ to determine $\dot{U}$ for given $U, \bar{x}, \dot{\bar{x}}$, and $t$. The solution $U(t)$ of $F(U(t), x(t), t)=0$ can be updated without iterative optimisation method by integrating (32) in real-time using continuation method [3]. In practical applications, $U(0)$ that satisfies $F(U(t), x(t), t)=0$ must be found through the numerical method and $\dot{\bar{x}}$ in (32) must be approximated by finite difference [35]. For further details about the C/GMRES, its error analysis, and proof see [35].

The next subsection presents the various inequality constraints handling methods. The proposal to utilise the FischerBurmeister (FB) function to handle inequality constraints for the C/GMRES algorithm is the contribution of the following subsection. 


\section{Inequality Constrains Handling Method}

Indirect methods are known to show fast numerical convergence in the neighbourhood of the optimal solution. However, handling of inequality constraints via PMP is in general non-trivial, due to the overall structure of the TP-BVP that depends on the sequence between singular/nonsingular and unconstrained/constrained arcs (if the respective constraint is active or not) as well as a prior knowledge of the OCP structure [36]. There are several works of literature to systematically transform a general inequality constrained OCP into a surrogate equality constrained OCP by various methods.

Let's consider the deterministic inequality constraint $g(x, u) \leq 0$ which the equivalent equality constraint $(C(x, u))$ based on the Auxiliary Variable method was proposed in [35]. The main idea is based on the introduction of an additional optimisation variables to transform inequality constraints into equality constraints as follows:

$$
C(x, u):=g(x, u)+\eta^{2}=0
$$

where $\eta \in \mathbb{R}^{s}$ denotes a vector of auxiliary variables which has to impose to (29) as optimisation variables. In order to avoid the singularity, a small dummy penalty is added to the cost function (25). For more details about inequality constraints handling using this method see e.g. [3], [28], and [35]. The main draw back in this method is that if the solution of the OCP is very close to the constraint boundary, the optimisation problem becomes ill-conditioned [37].

For the sake of simplicity, let's consider the nonlinear OCP (26) with deterministic constraints $\left(\beta_{j}=1\right)$. A part of the first-order necessary conditions for a solution to be optimal are based on Karush-Kuhn-Tucker (KKT) conditions which are also known as Nonlinear Complementarity Problem (NCP) given by:

$$
\begin{aligned}
& g_{j}\left(\hat{\bar{x}}^{*}\right) \leq 0, \\
& \mu_{j}^{*} \geq 0, \\
& \mu_{j}^{*} g_{j}\left(\hat{\bar{x}}^{*}\right)=0, \text { for all } j \in \mathbb{Z}_{[1, s]} .
\end{aligned}
$$

Conditions (34c) are also called complementary slackness conditions. It can be interpreted as if the $j^{\text {th }}$ inequality constraint of the primal problem is inactive at the optimum solution $g_{j}\left(\hat{\bar{x}}^{*}\right) \leq 0$, then the $j^{\text {th }}$ dual variable has to be zero $\left(\mu_{j}^{*}=0\right)$.

In order to account the complementary condition (34) in the necessary condition (28) and to avoid the ill-conditioning, a semi-smooth transformation is utilised in this paper. Two important and most widely used examples of complementarity functions are the natural residual function given by:

$$
\psi\left(\mu_{j}^{*}, g_{j}\left(\hat{\bar{x}}^{*}\right)\right)=\max \left\{\mu_{j}^{*}, g_{j}\left(\hat{\bar{x}}^{*}\right)\right\},
$$

and the FB function which is used in this paper as follows:

$$
\psi_{F B}\left(\mu_{j}^{*}, g_{j}\left(\hat{\bar{x}}^{*}\right)\right)=\sqrt{\mu_{j}^{*^{2}}, g_{j}\left(\hat{\bar{x}}^{*}\right)^{2}}-\left(\mu_{j}^{*}-g_{j}\left(\hat{\bar{x}}^{*}\right)\right) .
$$

Complementarity functions provide a convenient tool for converting problems that involve complementarity conditions into equations [38]. The complementarity conditions are satisfied if and only if the following condition using the FB function is satisfied:

$$
\psi_{F B}\left(\mu_{j}^{*}, g_{j}\left(\hat{\bar{x}}^{*}\right)\right)=0
$$

for each $j^{\text {th }}$ element of inequality constraints [39]. Therefore, the FB transformation converts the inequality constrained OCPs into an equivalent equality constrained OCP.

\section{E. Case Study: Energy Management for Extended Eco-ACC}

The state vector for the Extended Eco-ACC concept is defined as $x_{t}=\left[s_{h}, v_{h}, e_{h}\right]^{T} \in \mathbb{R}^{3}$; the control input is the traction input with the modelled delay of the power plant applied on the host vehicle as $u_{t}=u \in \mathbb{U} \subset \mathbb{R}$. The volatility of the preceding vehicle velocity and its position can be extremely wide, therefore regulating relative safe distance in an energy efficient method is of fundamental importance to the Extended Eco-ACC system. The measurable disturbance (e.g., Radar-based system) is defined as position $\left(s_{p}\right)$ and velocity $\left(v_{p}\right)$ of the preceding vehicles. Note that we refer the risk as the uncertainty related to the future values of relative distance $\bar{d}:=\bar{s}_{p}-\bar{s}_{h}$ and all states are measurable which the measurement noise is negligible. The disturbances as auxiliary states are concatenated with the system state vector to form the nominal state vector. From Eqs. (1), (2), (10), (11), and (12), the extended state vector is: $\bar{x}_{t}=\left[\dot{s}_{h}, \dot{v}_{h}, \dot{e}_{h}, \dot{\hat{s}}_{p}, \dot{\hat{v}}_{p}\right]^{T} \in \mathbb{R}^{5}$.

The cost-per-stage function for the Extended Eco-ACC system is defined as:

$V_{N}\left(x_{t}, \boldsymbol{\pi}\right):=\sum_{i=0}^{N-1}\left\|\hat{x}_{i}-x_{r e f}\right\|_{Q}^{2}+\left\|u_{i}-u_{r e f}\right\|_{R}^{2}+C \hat{x}_{i}^{T}$,

with corresponding weights $(Q, R, C)$. The control input is limited by:

$$
u_{\min }(v) \leq u \leq u_{\max }(v)
$$

where $u_{\min }(v)$ and $u_{\max }(v)$ can be identified based on the traction-velocity map of the BEV [28].

The state inequality constraints are lateral acceleration constraint as comfort level, speed limit constraint respecting the traffic regulation, relative distance constraint as safety constraint. In addition, a funnel constraint is introduced for the velocity of the host BEV as well as the energy consumption of the BEV should be limited to a certain level. The lateral acceleration of the host vehicle should be lower than the comfort level ( $\left.\Psi_{\text {ref }}\right)$ almost surely $\left(\beta_{1}=1\right)$ as follows:

$$
\operatorname{Pr}_{\Psi_{t}}\left[g_{1}\left(\hat{s}_{h_{i}}, \hat{v}_{h_{i}}\right):=\hat{v}_{h_{i}}^{2} / f_{c r v}\left(\delta\left(\hat{s}_{h_{i}}\right)\right) \leq \Psi_{\text {ref }}\right] \geq \beta_{1} \text {. }
$$

The velocity of the host vehicle almost surely $\left(\beta_{2}=1\right)$ should also be lower than speed limit zones as:

$$
\operatorname{Pr}_{s_{t}}\left[g_{2}\left(\hat{s}_{h_{i}}, \hat{v}_{h_{i}}\right):=\hat{v}_{h_{i}} \leq f_{l m t}\left(\hat{s}_{h_{i}}\right)\right] \geq \beta_{2}
$$

The spacing policy to define the safe strategy in following the preceding vehicle is based on Time-Headway (for more detail see e.g., [40]). The relative distance should be larger than the reference space $\left(d_{\text {ref }}:=d_{0}+v_{h} t_{h w}\right)$ with closed-loop $\beta_{3}$ confident level as follows:

$$
\operatorname{Pr}_{d_{t}}\left[g_{3}\left(\hat{d}_{i}\right):=d_{\text {ref }} \leq \hat{d}_{i}\right] \geq \beta_{3} .
$$

Furthermore, the velocity should be within the standstill and the reference set-point almost surely $\left(\beta_{4}=1\right)$ given by:

$$
\operatorname{Pr}_{v_{h_{t}}}\left[g_{4}\left(\hat{v}_{h_{i}}\right):=0 \leq \hat{v}_{h_{i}} \leq\left(v_{h_{r e f}}+v_{h_{r l x}}\right)\right] \geq \beta_{4},
$$


TABLE I

FEATURES OF THE SMPC, DNMPC, RSNMPC

\begin{tabular}{|c|c|c|c|}
\hline & $\begin{array}{c}\text { SMPC } \\
{[22]}\end{array}$ & $\begin{array}{c}\text { DNMPC } \\
{[3]}\end{array}$ & RSNMPC \\
\hline Road slope & - & $\checkmark$ & $\checkmark$ \\
\hline Curvatures & - & - & $\checkmark$ \\
\hline Speed Limit & - & - & $\checkmark$ \\
\hline $\begin{array}{c}\text { Risk } \\
\text { Sensitive }\end{array}$ & - & - & $\checkmark$ \\
\hline $\begin{array}{l}\text { Preceding } \\
\text { Vehicle } \\
\text { Model }\end{array}$ & $\begin{array}{l}\text { Markov } \\
\text { Chain }\end{array}$ & $\begin{array}{c}\text { Intuitive } \\
\text { Model }\end{array}$ & $\begin{array}{c}\text { Physical- } \\
\text { statistical } \\
\text { Model }\end{array}$ \\
\hline $\begin{array}{c}\text { Constraints } \\
\text { Handling } \\
\text { Method }\end{array}$ & $\begin{array}{c}\text { Implicit } \\
\text { Quadratic }\end{array}$ & $\begin{array}{l}\text { Implicit } \\
\text { Primal } \\
\text { Barrier }\end{array}$ & $\begin{array}{l}\text { Primal-dual } \\
\text { Fischer- } \\
\text { Burmeister }\end{array}$ \\
\hline
\end{tabular}

where $v_{h_{r e f}}$ is the reference set-point. The $v_{h_{r l x}}$ is the relaxed amount of velocity for the host vehicle to overspeed whenever it is required such as cruising a downhill scenario to take advantage of the gravity. The energy consumption of the BEV should be less than the permitted maximum amount almost surely $\left(\beta_{5}=1\right)$ as follows:

$$
\operatorname{Pr}_{v_{h_{t}}}\left[g_{5}\left(\hat{e}_{h_{i}}\right):=\hat{e}_{h_{i}} \leq\left(e_{h_{r e f}}+e_{h_{r l x}}\right)\right] \geq \beta_{5},
$$

where $e_{h_{r e f}}$ and $e_{h_{r l x}}$ are the reference energy consumption and its relaxed value, respectively.

Table I shows a brief features of the proposed RSNMPC in comparison with the mentioned state-of-the-art methods for the case study. The proposed RSNMPC is compared with the deterministic NMPC (DNMPC) introduced in [3] and the SMPC presented in [22].

\section{System Evaluation}

The proposed Extended Eco-ACC system has been evaluated with numerical simulations using realistic values of the parameters and practical field experiments on the test track. Obtained results demonstrate the effectiveness of the proposed method for a semi-autonomous BEV in terms of safe and energy-efficient states regulation and constraints satisfaction.

\section{A. Simulation Results}

A third generation Smart-ED commercial BEV is available for simulation and practical experiments. The parameters of the Smart-ED dynamics model are derived from data sheets and field measurements as $m=975 \mathrm{~kg}, \delta_{1}=0.04$, $\delta_{2}=0.0025, i_{g}=9.922: 1, \rho=1.2041 \mathrm{~kg} / \mathrm{m}^{3}$, $A_{f}=2.057 \mathrm{~m}^{2}, C_{D 0}=0.35$, and $g=9.81 \mathrm{~m} / \mathrm{s}^{2}$. The main specifications of the Smart-ED are summarised in [13] and [14]. A dynamometer test has been conducted for the energy consumption model parameters, (10) which is identified as $a_{2}=0.01622, a_{1}=0.244, a_{0}=1.129, b_{3}=0, b_{2}=0.02925$, $b_{1}=0.257$, and $b_{0}=1.821$ with $98.46 \%$ coefficient of determination (R-squared) [28]. The prediction horizon for the predictive controller is set to $T=10 \mathrm{~s}$ to cover upcoming road geometry, traffic speed limit zones and the preceding vehicle motion prediction with $N=20$ discretized steps.

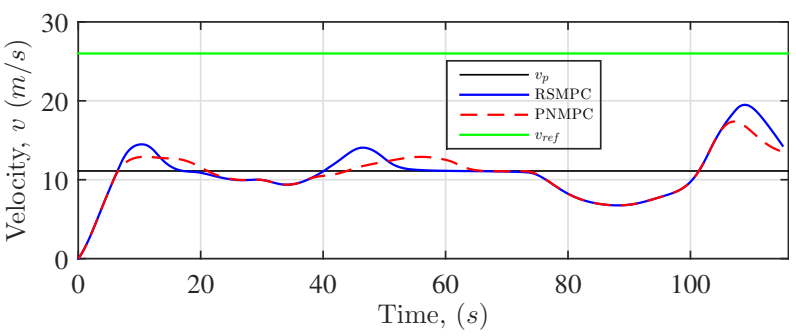

(a)

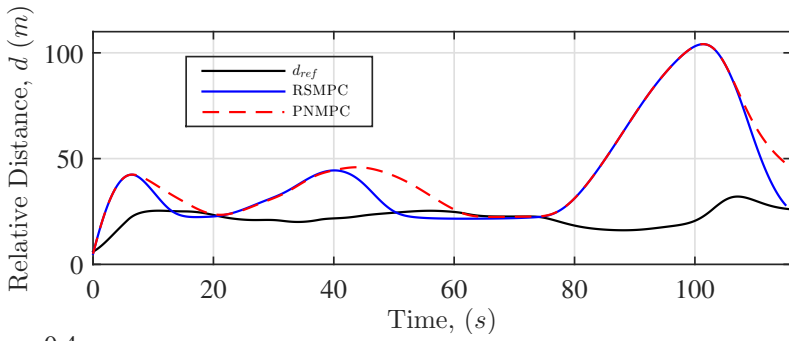

(b)

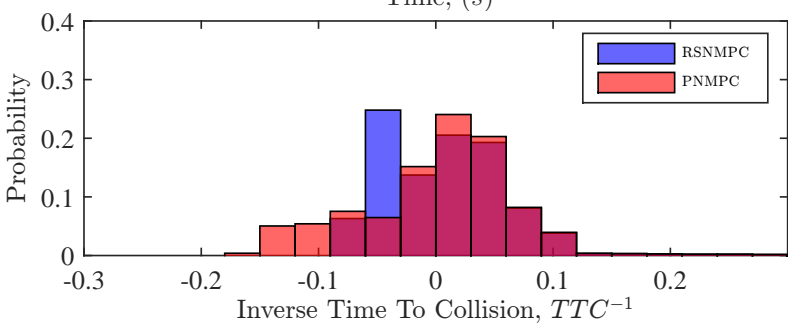

Fig. 5. Performance of RSNMPC vs. PNMPC for (a) Velocity regulation, (b) Relative distance regulation, and (c) Inverse Time To Collision $\left(T T C^{-1}\right)$

Fig. 5 shows the simulation results of the proposed RSNMPC for the optimal energy management of the BEV on a realistic hilly and curvy road of the test track. In this setup, the BEV follows the preceding vehicle with the close spacing setting as $d_{0}=6 \mathrm{~m}$ and $t_{h w}=1.5 \mathrm{~s}$ which could improve traffic flaw microscopically. Performance of the RSNMPC is compared with a Perfect NMPC (PNMPC) which the uncertainty of the preceding vehicle is exactly known in advance along the prediction horizon.

Fig. 5a shows the BEV velocity profile. The BEV speeds up to until the first and second curves $(20 \leq t \leq 40)$ where it has to slow down where the lateral acceleration constraint should be satisfied. As it is shown, the RSNMPC is faster than the PNMPC controller due to lack of knowledge from the preceding vehicle behaviour and assuming that the preceding vehicle will speed up. However, during the first and second curves, the RSNMPC and PNMPC show similar behaviour due to more accurate prediction. Fig. $5 \mathrm{~b}$ shows the relative distance regulation performance where the RSNMPC is more aggressive than PNMPC in this part of the test track. This is due to the constant velocity profile of the preceding vehicle with perfect measurement in a simulation environment which leads to low variance estimation in relative distance measurement. Therefore, the EVaR evaluation cause the chance constraint (42) to be treated almost surely. Afterwards, the controllers increase velocity again up to the point where the third and fourth curves are in its prediction horizon $(83 \leq t \leq 109)$ where both controllers slow down to fulfil the relative distance and the lateral acceleration constraints on curves. Since the RSNMPC is not aware of the future realised velocity profile 
of the preceding vehicle, it shows less optimum behaviour in comparison to the PNMPC. However, the RSNMPC shows similar behaviour close to the PNMPC performance within $66 \leq t \leq 106$. Finally, both controllers speed up once more to reach the starting point while satisfying the relative distance safety constraint. Fig. 5c shows the performance of the RSNMPC in comparison to PNMPC in terms of Inverse of Time To Collision $\left(T T C^{-1}:=\frac{v_{p}-v_{h}}{d}\right)$ probability distribution. The $T T C^{-1}$ is a direct and continuous indicator for the collision risk. The lower values indicate the more dangerous situations while zero implies the preserving trend. The RSNMPC shows sharper velocity and relative distance regulations which could increase its energy consumption. However, due to the statistically accurate prediction model of the preceding vehicle and considering the upcoming road geometries with energy consumption map of the Smart-ED, the RSNMPC is approximately $+89 \%$ as energy-efficient as the PNMPC on the test track despite unknown preceding vehicle behaviour.

In order to demonstrate the performance enhancements and compare the proposed approach with the mentioned state-ofthe-art methods in a fair and informative manner, the European Urban Driving Cycle (EUDC) is used to represent the preceding vehicle velocity profile $\left(v_{p}\right)$. The proposed RSNMPC is compared with the deterministic NMPC (DNMPC) introduced in [3], the SMPC presented in [22], and distributionally Robust SNMPC (RNMPC) presented in [25] which is configured with worst case scenario. The cruising velocity reference is fixed to $v_{h_{\text {ref }}}=26 \mathrm{~m} / \mathrm{s}$ for all of the controllers with the same values for $d_{0}=4 \mathrm{~m}$ and $t_{h w}=3 \mathrm{~s}$ considered in [22].

Fig. 6a shows the performance of different controllers in terms of velocity regulations. The DNMPC, RNMPC, and RSNMPC track the preceding vehicle and cruising reference with less overshoot compared to the SMPC. The proposed RSNMPC benefits from the closed-loop inequality constraints handling method, compared to the DNMPC using the conventional soft constrained penalty method as well as the SMPC using the quadratic cost function to handle the relative distance inequality. Fig. 6b and Fig. 6c show the relative distance regulation performance and related histogram information around the violation region. The DNMPC hardly minimises the constraint violation while the SMPC regulates the relative distance irrespective of the violation of the reference tracking. The RNMPC shows a too conservative behaviour, where the RSNMPC satisfies the chance constraint performance requirement. Note that in Fig. $6 \mathrm{c}$, the positive values denote the constraint satisfaction while the negative values represent the constraint violation. The OCP calculation time for the proposed RSNMPC is $5.3 \mathrm{~ms}$, compared to the SMPC with $1 \mathrm{~s}$; the RNMPC with $3.5 \mathrm{~m}$, and the DNMPC with $2.2 \mathrm{~ms}$. Although the DNMPC is faster than the RSNMPC and RNMPC, it has suffered from the low fidelity preceding vehicle motion model. Furthermore, the more steady velocity profile with proper constraints satisfaction which is generated by the RSNMPC provides a better drive comfort with lower energy consumption. Energy consumption and average computation time of the OCP can be concluded from Table II. The proposed RSNMPC is approximately $+1 \%$ more energy

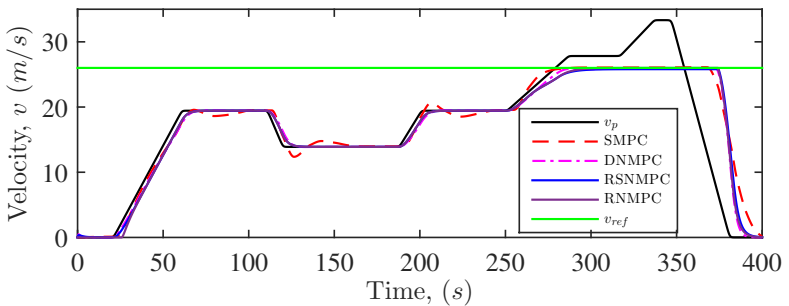

(a)

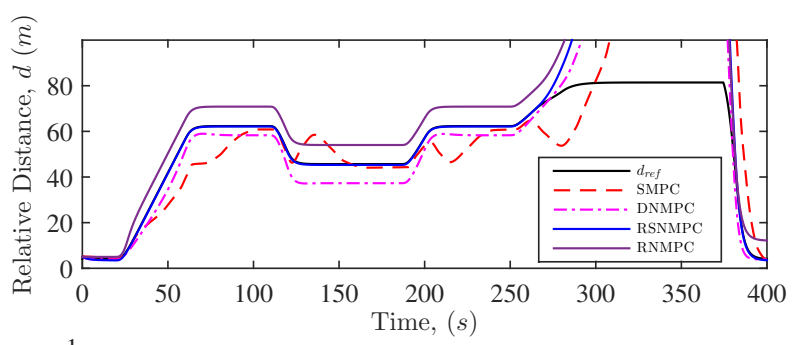

(b)

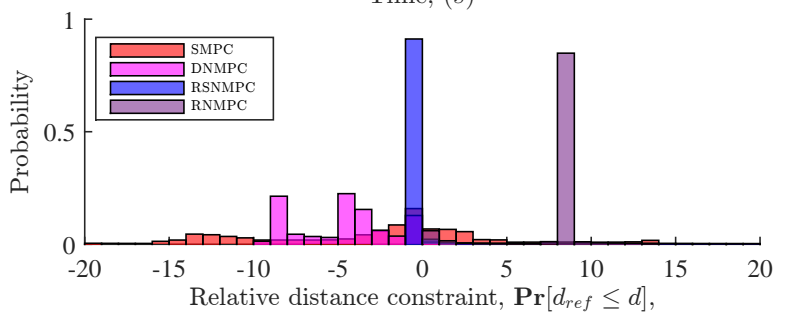

Fig. 6. Performance of controllers for (a) Velocity and (b) Relative distance regulations, with (c) probability distribution of chance constraint around violation region

TABLE II

ENERGY CONSUMPTION AND COMPUTATION TIME OF OCPS

\begin{tabular}{c|c|c|c|c} 
& SMPC & DNMPC & RNMPC & RSNMPC \\
\hline Eco. $(\mathrm{kWh})$ & - & 1.4778 & 1.4770 & 1.4680 \\
\hline OCP $(\mathrm{ms})$ & 1000 & 2.2 & 3.5 & 5.3
\end{tabular}

efficient than the DNMPC method, thanks to proper relative distance regulation. In the carried out simulations, the road is assumed to be flat and straight with no speed limit zones. Thus, there are few potentials to save energy which is achieved by accounting the energy consumption dynamics. In other words, for longer trips with more hilly and curvy roads, the proposed method has higher potential to save energy. The next subsection confirms the claim in field experimental tests.

\section{B. Field Experimental Results}

In order to validate the proposed concept, the RSNMPC is experimentally implemented on the Smart-ED BEV and a city vehicle Peugeot 108 is chosen to represent the preceding vehicle. The Extended Eco-ACC system is tested on the closed track (Fig. 7).

The position of the host BEV is updated by the Global Positioning System (GPS) sensor. The velocity and energy consumption of the vehicle including the battery current and voltage information is updated by the Controller Area Network (CAN-bus) through the On-Board Diagnose (OBD) interface. A $77 \mathrm{GHz}$ Electronically Scanning Radar (ESR) is installed on the $\mathrm{BEV}$ to measure the position and velocity of the preceding vehicle (Fig. 8). The onboard computational resource for 


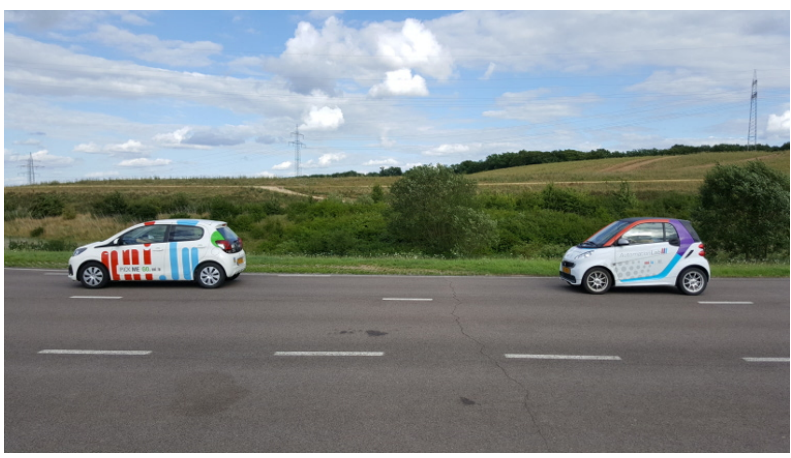

Fig. 7. Extended Eco-ACC System for Semi-autonomous BEV on Test Track

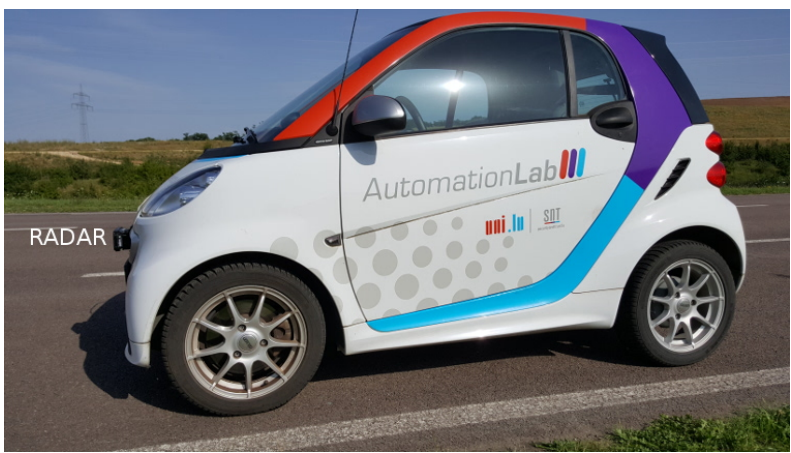

Fig. 8. Installed Electronically Scanning Radar (ESR) on Smart-ED

the Extended Eco-ACC system is foreseen by the Robot Operating System (ROS) on the Intel ${ }^{\circledR}$ Core $^{\mathrm{TM}}$ i7 with a memory of $7.7 \mathrm{GiB}$ PC and connection panel. The connection panel is developed for the system power supply and actuators communication (Fig. 9).

The control input of the proposed RSNMPC is realised by actuating either the accelerator pedal or brake actuator. The accelerator pedal is replaced by an electronic board (Eaccelerator) to manipulate the required acceleration and to imitate the electric signals generated by the original accelerator pedal of the Smart-ED. The brake actuator is manipulated by an electric stepper motor that is connected to the brake pedal by a planetary gearbox and flexible cable. The automatic brake actuation is designed in a way that preserves the possibility for the driver to brake in emergency cases. Fig. 10 shows the configuration of the E-accelerator and brake actuators for the Extended Eco-ACC system.

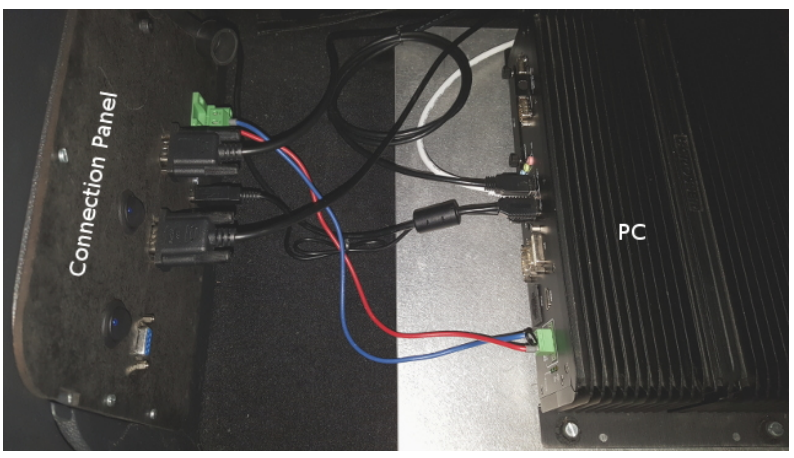

Fig. 9. Robot Operating System (ROS) operated PC with Connection Panel

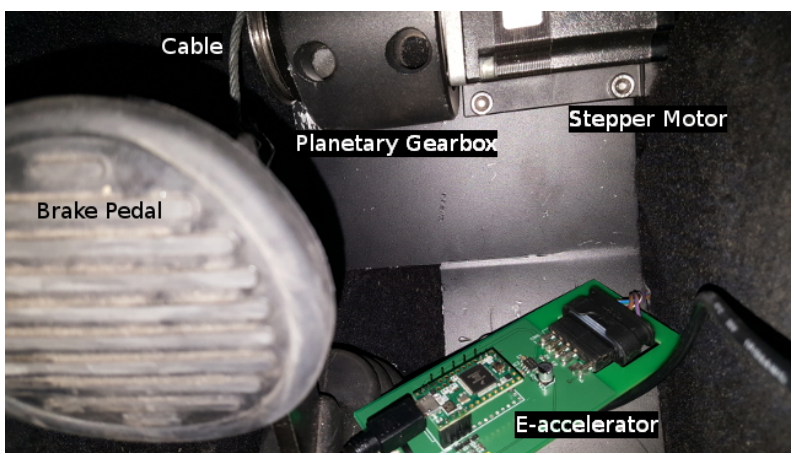

Fig. 10. Automatic E-Accelerator and Brake Actuators

(a)
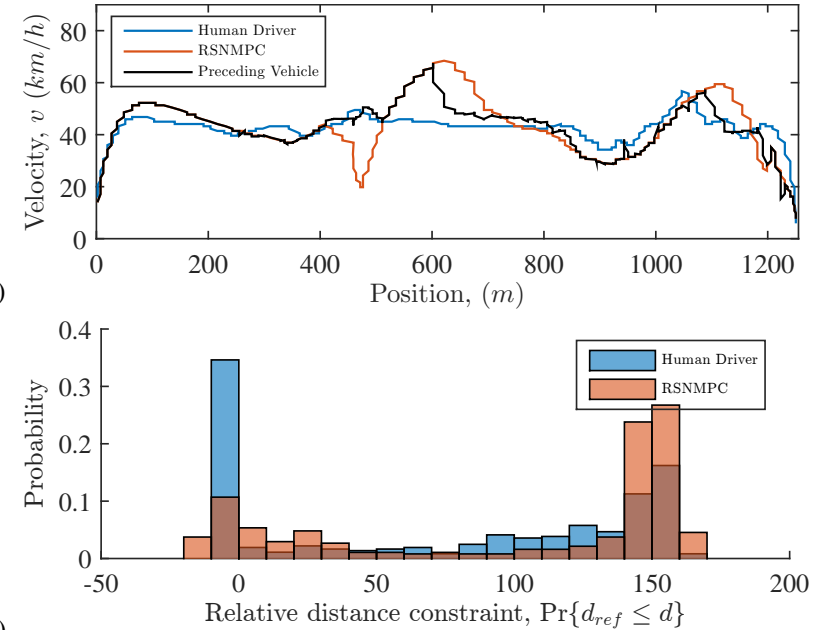

(b)

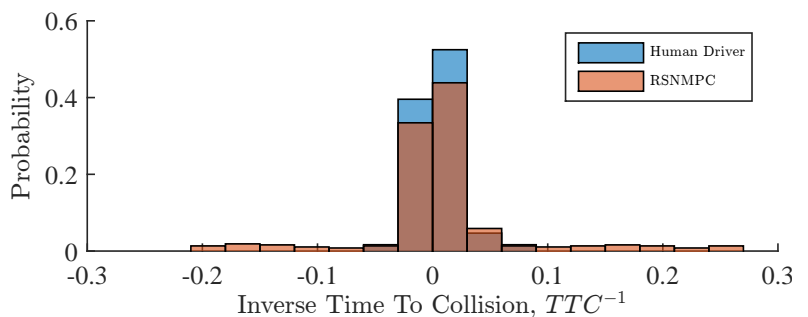

(c)

Fig. 11. Performance of RSNMPC vs Human Driver for (a) Velocity regulation, (b) Probability distribution of relative distance, and (c) Inverse Time To Collision $\left(T T C^{-1}\right)$

The reference velocity is fixed to maximum $v_{h_{\text {ref }}}=$ $100 \mathrm{~km} / \mathrm{h}$ with spacing setting $d_{0}=6 \mathrm{~m}$ and $t_{h w}=1.5 \mathrm{~s}$. The human driver of the preceding vehicle is cruising at $v_{p}=50 \mathrm{~km} / \mathrm{h}$ as often as possible. Fig. 11a shows the performance of the human driver in comparison to the RSNMPC in terms of velocity regulations. Fig. $11 \mathrm{~b}$ and Fig. 11c demonstrate the performance of relative distance regulation and its $T T C^{-1}$ as risk of rear-end collision, respectively. The relative distance chance constraint is satisfied with minimum violation in comparison to the human driver.

Fig. 12a shows the performance of power consumption by the human driver and the RSNMPC. It is shown that the variance of the power consumption by the RSNMPC is lower than the one of the human driver for similar situations which lead to approximately $+21 \%$ more energy efficiency in comparison to the human driver. Fig. 12b and Fig. 12c demonstrate the performance of the actuated E-accelerator and 
(a)
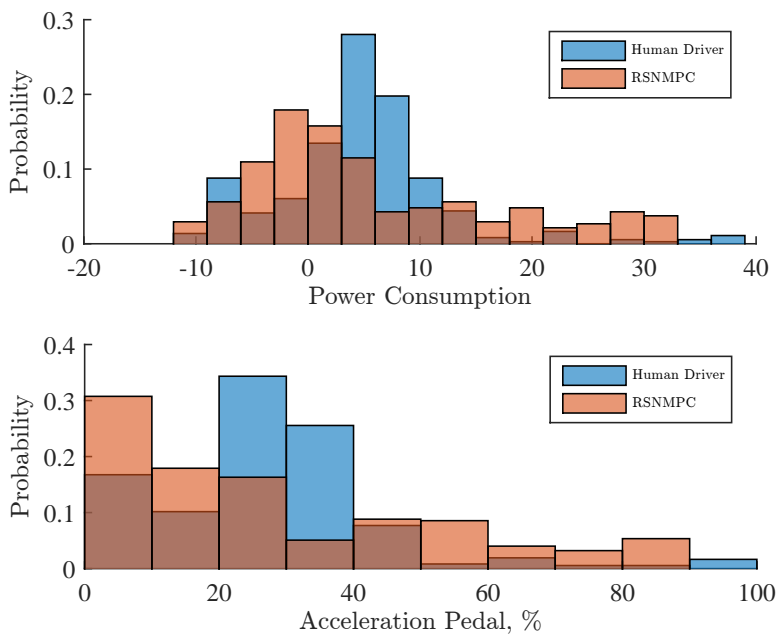

(b)

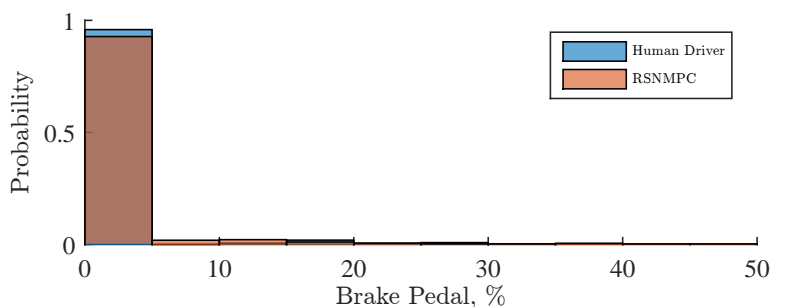

(c)

Fig. 12. Performance of RSNMPC vs Human Driver for (a) Probability distribution of Power consumption, (b) E-accelerator, and (c) Brake pedal actuations

brake pedal, respectively. It is shown that the RSNMPC has applied a lower amount of acceleration and relatively similar brake actuation in comparison to the human driver which improves the BEV energy consumption.

In this paper we have evaluated field experiments of the cut-in and cut-out scenarios in order to demonstrate the performance of the RSNMPC for unforeseen situations such as cut-in, cut-out or Emergency Braking (EB) (for more details see [41]). In this practical test, the $\mathrm{BEV}$ is cruising along the track while the preceding vehicle cuts-in the lane of the BEV after having overtaken it. Fig. 13 shows the velocity and relative distance regulations, receptively. It is shown that the RSNMPC can manage the unexpected cut-in situation. Fig. 13 a shows a smooth reduction in velocity of the BEV to adapt to the preceding vehicle. Furthermore, Fig. 13 demonstrates the relative distance regulation with the reference relative distance to preserve a safe distance to the preceding vehicle.

In addition, the cut-out test scenario is carried out to demonstrate the performance of the RSNMPC for the unforeseen situation. In this practical test, the BEV is cruising in a carfollowing situation. The preceding vehicle cuts-out the driving lane of the BEV. Fig. 14 shows the velocity and relative distance regulations, receptively. It is shown that the RSNMPC is able to handle the unexpected cut-out situation. Fig. 14a shows a smooth increase in velocity of the BEV to reach the desired velocity after the cut-out situation. The relative distance after the cut-out event reach to the maximum value indicating the free-flowing condition. (a)

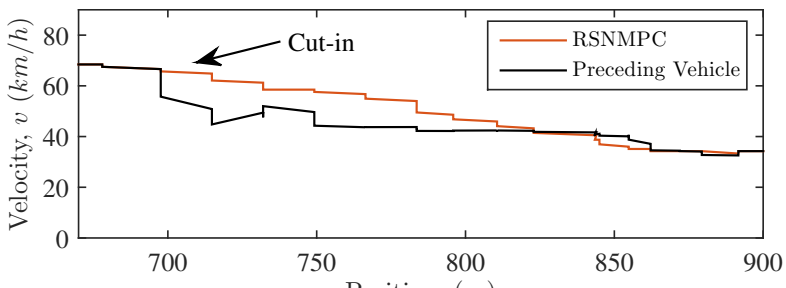

(b)

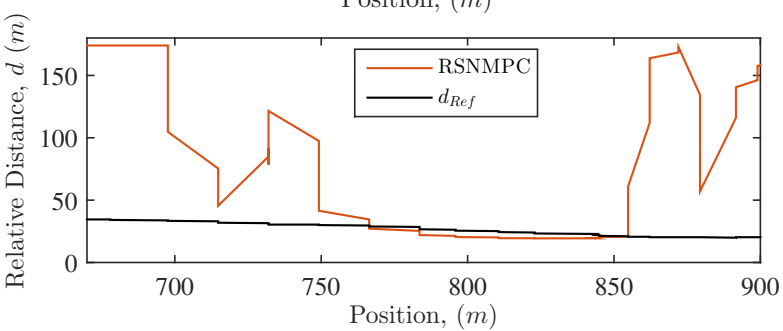

Fig. 13. Performance of RSNMPC in Cut-in scenario for (a) Velocity and (b) Relative distance regulations

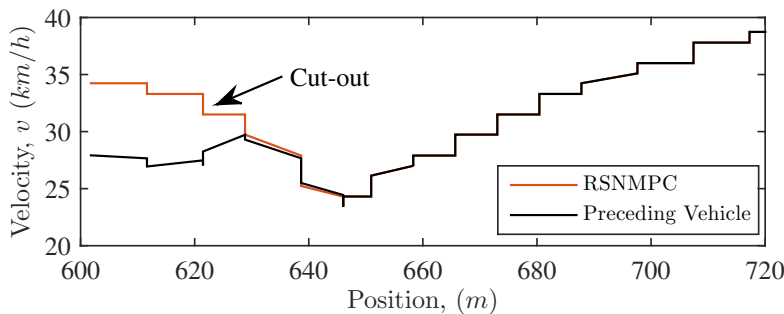

(a)

(b)

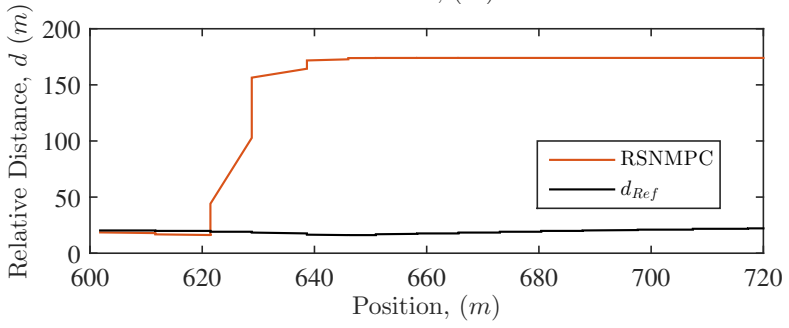

Fig. 14. Performance of RSNMPC in Cut-out scenario for (a) Velocity and (b) Relative distance regulations

\section{CONClusion ANd Future RESEARCH}

A real-time risk-sensitive nonlinear model predictive controller for optimal energy management of an electric vehicle has been proposed in this paper. The system accounts for upcoming road slopes, curves, speed limit zones, as well as uncertainty in the preceding vehicle behaviour to determine the optimal efficient drive strategy in an anticipated manner. Optimal energy consumption based on a semi-autonomous ecological advanced driver assistance system has been designed to improve the longitudinal velocity regulation in a safe and energy-efficient driving manner. The computation time for the proposed stochastic nonlinear predictive controller was found to be a real-time algorithm by using a closed-loop coherent risk measure to quantify the risk involved in the chance constraints. Obtained simulation and field experimental tests have been evaluated and compared with state-of-the-art methods as well as a human driver. The energy efficiency of the risk-sensitive predictive control is found to be approximately $+21 \%$ more energy efficient in comparison to the human driver in similar situations. The performance of the proposed method has shown significant improvement in safety and energy efficiency which 
extend the limited cruising range of the electric vehicle. Further practical experiments will be conducted to validate the proposed method in more complex scenarios. In addition, extending the perception capability of the proposed system with a vision system or connected vehicles technology has high potential to improve the overall system performance.

\section{ACKNOWLEDGMENT}

This work is supported by the FNR "Fonds national de la Recherche" (Luxembourg) through the AFR "Aides à la Formation-Recherche" Ph.D. grant scheme No. 7041503. The authors would like to acknowledge the FNR, Delphi Automotive Systems S.A., Centre de Formation pour Conducteurs S.A., T. Schwickart, and Remi Radinovic for their support and valuable comments in this study.

\section{REFERENCES}

[1] M. Ehsani, Y. Gao, and A. Emadi, Modern Electric, Hybrid Electric, and Fuel Cell Vehicles: Fundamentals, Theory, and Design, 2nd ed. CRC Press, 2009.

[2] M. Helmbrecht, C. Olaverri-Monreal, K. Bengler, R. Vilimek, and A. Keinath, "How electric vehicles affect driving behavioral patterns," IEEE Intelligent Transportation Systems Magazine, vol. 6, no. 3, pp. 22-32, 2014.

[3] M. A. S. Kamal, M. Mukai, J. Murata, and T. Kawabe, "Model Predictive Control of Vehicles on Urban Roads for Improved Fuel Economy," IEEE Transactions on Control Systems Technology, vol. 21, no. 3, pp. 831841, may 2013.

[4] J. Y. Yong, V. K. Ramachandaramurthy, K. M. Tan, and N. Mithulananthan, "A review on the state-of-the-art technologies of electric vehicle, its impacts and prospects," Renewable and Sustainable Energy Reviews, vol. 49, pp. 365-385, sep 2015.

[5] A. Sciarretta, G. De Nunzio, and L. L. Ojeda, "Optimal Ecodriving Control: Energy-Efficient Driving of Road Vehicles as an Optimal Control Problem," IEEE Control Systems, vol. 35, no. 5, pp. 71-90, oct 2015.

[6] Q. Jin, G. Wu, K. Boriboonsomsin, and M. J. Barth, "Power-Based Optimal Longitudinal Control for a Connected Eco-Driving System," IEEE Transactions on Intelligent Transportation Systems, vol. 17, no. 10, pp. 2900-2910, oct 2016.

[7] C. P. Rommerskirchen, M. Helmbrecht, and K. J. Bengler, "The Impact of an Anticipatory Eco-Driver Assistant System in Different Complex Driving Situations on the Driver Behavior," IEEE Intelligent Transportation Systems Magazine, vol. 6, no. 2, pp. 45-56, 2014.

[8] D. Q. Mayne, "Model predictive control: Recent developments and future promise," Automatica, vol. 50, no. 12, pp. 2967-2986, dec 2014.

[9] J. Han, A. Sciarretta, L. Leon Ojeda, G. De Nunzio, and L. Thibault, "Safe- and eco-driving control for connected and automated electric vehicles using analytical state-constrained optimal solution," IEEE Transactions on Intelligent Vehicles, vol. 8858, no. c, pp. 1-1, 2018.

[10] T. Gindele, S. Brechtel, and R. Dillmann, "Learning Driver Behavior Models from Traffic Observations for Decision Making and Planning," IEEE Intelligent Transportation Systems Magazine, vol. 7, no. 1, pp. 69-79, 2015.

[11] M. A. S. Kamal, S. Taguchi, and T. Yoshimura, "Efficient Driving on Multilane Roads Under a Connected Vehicle Environment," IEEE Transactions on Intelligent Transportation Systems, vol. 17, no. 9, pp. 2541-2551, sep 2016.

[12] K. Bengler, K. Dietmayer, B. Farber, M. Maurer, C. Stiller, and H. Winner, "Three decades of driver assistance systems: Review and future perspectives," IEEE Intelligent Transportation Systems Magazine, vol. 6, no. 4, pp. 6-22, 2014.

[13] T. Schwickart, H. Voos, J.-R. Hadji-Minaglou, M. Darouach, and A. Rosich, "Design and simulation of a real-time implementable energyefficient model-predictive cruise controller for electric vehicles," Journal of the Franklin Institute, vol. 352, no. 2, pp. 603-625, feb 2015.

[14] T. Schwickart, H. Voos, J.-R. Hadji-Minaglou, and M. Darouach, "A Fast Model-Predictive Speed Controller for Minimised Charge Consumption of Electric Vehicles," Asian Journal of Control, vol. 18, no. 1, pp. 133149, jan 2016
[15] S. E. Li, Z. Jia, K. Li, and B. Cheng, "Fast Online Computation of a Model Predictive Controller and Its Application to Fuel EconomyOriented Adaptive Cruise Control," IEEE Transactions on Intelligent Transportation Systems, vol. 16, no. 3, pp. 1199-1209, jun 2015.

[16] L. Grüne and J. Pannek, Nonlinear Model Predictive Control, ser. Communications and Control Engineering. London: Springer London, 2011, vol. 1.

[17] S. E. Li, Q. Guo, S. Xu, J. Duan, S. Li, C. Li, and K. Su, "Performance Enhanced Predictive Control for Adaptive Cruise Control System Considering Road Elevation Information," IEEE Transactions on Intelligent Vehicles, vol. 2, no. 3, pp. 150-160, sep 2017.

[18] M. Vajedi and N. L. Azad, "Ecological Adaptive Cruise Controller for Plug-In Hybrid Electric Vehicles Using Nonlinear Model Predictive Control," IEEE Transactions on Intelligent Transportation Systems, vol. 17, no. 1, pp. 113-122, jan 2016.

[19] J. B. Rawlings and D. Q. Mayne, Model Predictive Control: Theory and Design. Nob Hill Publishing, 2012

[20] C. M. Filho, M. H. Terra, and D. F. Wolf, "Safe Optimization of Highway Traffic With Robust Model Predictive Control-Based Cooperative Adaptive Cruise Control," IEEE Transactions on Intelligent Transportation Systems, pp. 1-11, 2017.

[21] A. Mesbah, "Stochastic Model Predictive Control: An Overview and Perspectives for Future Research," IEEE Control Systems, vol. 36, no. 6, pp. 30-44, dec 2016.

[22] M. Bichi, G. Ripaccioli, S. Di Cairano, D. Bernardini, A. Bemporad, and I. Kolmanovsky, "Stochastic model predictive control with driver behavior learning for improved powertrain control," in 49th IEEE Conference on Decision and Control (CDC). IEEE, dec 2010, pp. 6077-6082.

[23] D. Moser, H. Waschl, H. Kirchsteiger, R. Schmied, and L. del Re, "Cooperative adaptive cruise control applying stochastic linear model predictive control strategies," in 2015 European Control Conference (ECC). IEEE, jul 2015, pp. 3383-3388.

[24] Y. Zhou, S. Ahn, M. Chitturi, and D. A. Noyce, "Rolling horizon stochastic optimal control strategy for ACC and CACC under uncertainty," Transportation Research Part C: Emerging Technologies, vol. 83, pp. 61-76, oct 2017.

[25] S. A. Sajadi-Alamdari, H. Voos, and M. Darouach, "Fast stochastic nonlinear model predictive control for electric vehicle advanced driver assistance systems," in 2017 IEEE International Conference on Vehicular Electronics and Safety (ICVES). IEEE, jun 2017, pp. 91-96.

[26] S. A. Sajadi-Alamdari, H. Voos, and M. Darouach, "Risk-averse Stochastic Nonlinear Model Predictive Control for Real-time Safety-critical Systems," IFAC-PapersOnLine, vol. 50, no. 1, pp. 5991-5997, jul 2017.

[27] S. Watkins and G. Vino, "The effect of vehicle spacing on the aerodynamics of a representative car shape," Journal of Wind Engineering and Industrial Aerodynamics, vol. 96, no. 6-7, pp. 1232-1239, jun 2008.

[28] S. A. Sajadi-Alamdari, H. Voos, and M. Darouach, "Nonlinear model predictive extended eco-cruise control for battery electric vehicles," in 2016 24th Mediterranean Conference on Control and Automation (MED). IEEE, jun 2016, pp. 467-472.

[29] D. S. Turne, J. Peter M. Briglia, and K. Fitzpatrick, "Modeling Operating Speed: Synthesis Report," Washington, DC, Tech. Rep. July, 2011.

[30] "Centre de Formation pour Conducteurs." [Online]. Available: http: //www.cfc.lu/

[31] X. Yang and J. Maciejowski, "Risk-Sensitive Model Predictive Control with Gaussian Process Models," in IFAC-PapersOnLine, vol. 48, no. 28. Elsevier B.V., 2015, pp. 374-379.

[32] J. Gonzalez and J. Moriarty, "Risk-sensitive optimal switching and applications to district energy systems," in 2014 International Conference on Probabilistic Methods Applied to Power Systems (PMAPS). IEEE, jul 2014, pp. 1-6.

[33] A. Ahmadi-Javid, "Entropic Value-at-Risk: A New Coherent Risk Measure," Journal of Optimization Theory and Applications, vol. 155, no. 3, pp. 1105-1123, dec 2012.

[34] B. Einarsson, Accuracy and Reliability in Scientific Computing, B. Einarsson, Ed. Society for Industrial and Applied Mathematics, jan 2005.

[35] T. Ohtsuka, "A continuation/GMRES method for fast computation of nonlinear receding horizon control," Automatica, vol. 40, no. 4, pp. 563574, apr 2004.

[36] K. Graichen, A. Kugi, N. Petit, and F. Chaplais, "Handling constraints in optimal control with saturation functions and system extension," Systems \& Control Letters, vol. 59, no. 11, pp. 671-679, nov 2010.

[37] J. M. Maciejowski, Predictive Control: With Constraints. Prentice Hall, 2002, no. Pearson Education. 
[38] A. F. Izmailov and M. V. Solodov, Newton-Type Methods for Optimization and Variational Problems, ser. Springer Series in Operations Research and Financial Engineering. Cham: Springer International Publishing, 2014.

[39] M. Huang, H. Nakada, K. Butts, and I. Kolmanovsky, "Nonlinear Model Predictive Control of a Diesel Engine Air Path: A Comparison of Constraint Handling and Computational Strategies," IFAC-PapersOnLine, vol. 48, no. 23, pp. 372-379, 2015.

[40] A. Eskandarian, Ed., Handbook of Intelligent Vehicles. London: Springer London, 2012, vol. 2.

[41] S. A. Sajadi-Alamdari, H. Voos, and M. Darouach, "Stochastic Model Predictive Control for Eco-Driving Assistance Systems in Electric Vehicles," 2017. [Online]. Available: https://www.youtube.com/watch? $\mathrm{v}=9$ sAopTHayZE

\section{About the Authors}

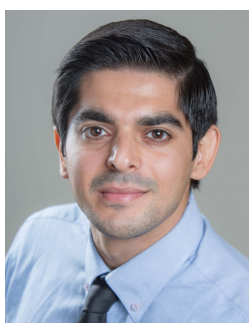

Seyed Amin Sajadi-Alamdari received a B.Eng. in Computer Hardware from Shomal University and a M.Eng. in Mechatronics Engineering from Qazvin Azad University, Iran in 2007 and 2011 respectively. In 2014 he joined the Interdisciplinary Centre for Security, Reliability and Trust (SnT) at the University of Luxembourg as a PhD student. His research interests are stochastic optimisation, nonlinear model predictive control, real-time systems, advanced driver assistance systems and semi/autonomous driving for electric vehicles.

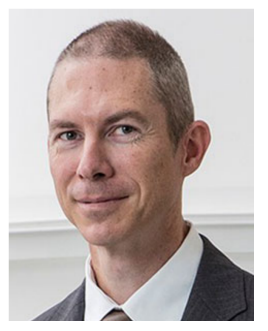

Holger Voos studied Electrical Engineering at the Saarland University and received the Doctoral Degree in Automatic Control from the Technical University of Kaiserslautern, Germany, in 2002. From 2000 to 2004, he was with Bodenseewerk Gertetechnik GmbH, Germany as Systems Engineer in aerospace and robotics. From 2004 to 2010, he was Professor at the University of Applied Sciences Ravensburg-Weingarten, Germany, and the head of the Mobile Robotics Lab there. Since 2010, he is Professor at the University of Luxembourg in the Faculty of Science, Technology and Communication, Research Unit of Engineering Sciences. He is the head of the Automatic Control Research Group and also the head of the Automation Lab in the Interdisciplinary Centre of Security, Reliability and Trust (SnT) at the University of Luxembourg. His research interests are in the area of distributed and networked control, model predictive control and safe and secure automation systems with applications in mobile robotics, energy systems and biomedicine.

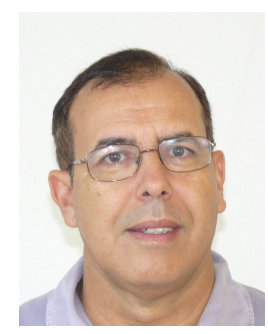

Mohamed Darouach graduated from "Ecole Mohammadia d'Ingénieurs", Rabat, Morocco, in 1978, and received the Doctor Engineer and Doctor of Sciences degrees from Nancy University, France, in 1983 and 1986, respectively. From 1978 to 1986 he was Associate Professor and Professor of automatic control at Ecole Hassania des Travaux Publics, Casablanca, Morocco. Since 1987 he is Professor at the Université de Lorraine. He was Vice Director of the Research Center in Automatic Control of Nancy (CRAN UMR 7039, Nancy-University, CNRS) from 2005 to 2013. He obtained a degree Honoris Causa from the Technical University of IASI and since 2010 he is a member of the Scientific council of the University of Luxembourg. Since July 2013, he is Vice Director of the University Institute of Technology of Longwy (Université de Lorraine). He held invited positions at the University of Alberta, Edmonton. His research interests span theoretical control, observers design, and control of large-scale uncertain systems with applications. 